\title{
ERGODIC PROPERTIES OF MARKOV SEMIGROUPS IN VON NEUMANN ALGEBRAS
}

\author{
Katarzyna Kielanowicz and Andrzej ŁucZaK
}

\begin{abstract}
We investigate ergodic properties of Markov semigroups in von Neumann algebras with the help of the notion of constrictor, which expresses the idea of closeness of the orbits of the semigroup to some set, as well as the notion of 'generalised averages', which generalises to arbitrary abelian semigroups the classical notions of Cesàro, Borel, or Abel means. In particular, mean ergodicity, asymptotic stability, and structure properties of the fixed-point space are analysed in some detail.
\end{abstract}

2010 Mathematics Subject Classification: Primary: 46L55; Secondary: 47A35.

Key words: ergodic theorems, Markov semigroups, positive maps, von Neumann algebra.

\section{Introduction}

The aim of this paper is to investigate ergodic properties of Markov semigroups acting on the predual of a von Neumann algebra. Our analysis has three main parts. In the first part, we consider the notion of mean ergodicity, show that our definition is equivalent to customary definitions known in some concrete cases, and give a number of criteria for a Markov semigroup to be mean ergodic. In the second part, we make use of the notion of constrictor introduced to ergodic theory in [21] and $[\mathbf{2 2}]$, and then considered in several papers. This notion expresses the idea of closeness of the orbits of the semigroup to some set. We show that the existence of a compact constrictor sufficiently close to the orbits leads to mean ergodicity of the semigroup as well as to interesting structure properties of the fixed-point space of the (dual) semigroup. Moreover, if this constrictor is 'sufficiently small', then the semigroup is asymptotically stable - a property which is also known as 'approach to equilibrium', and which was studied in many papers. In the third part, we consider generalised averages which are counterparts for arbitrary abelian semigroups of the classical Cesàro, Borel, or Abel means. We show that the mean ergodicity of the semigroup, the structure of the fixed-point space, or asymptotic stability follow from some natural properties of these averages. 
In our approach, we avoid the common assumption of complete positivity of the dual semigroup. Instead, we separate out further implications of strong positivity in our conclusions. Also, the continuity of the action of the semigroup turns out to be immaterial in most cases (with the exception of Theorem 19) which allows us to consider an arbitrary abelian semigroup with discrete topology, thereby generalising the customary approach in which the semigroup is indexed either by nonnegative integers or by nonnegative real numbers.

An extensive overview of positive semigroups on the predual of a von Neumann algebra is contained in [5, Section 3.3].

\section{Preliminaries and notation}

Let $\mathcal{M}$ be an arbitrary von Neumann algebra with predual $\mathcal{M}_{*}$, acting on a Hilbert space $\mathcal{H}$. The selfadjoint part of $\mathcal{M}$ will be denoted by $\mathcal{M}^{h}$, and $\mathcal{M}^{+}$will stand for the positive part of $\mathcal{M}$. The same notational convention applies to subspaces of $\mathcal{M}$, as well as to $\mathcal{M}_{*}$ and its subspaces. For any Banach space $X$, we will denote by $X_{1}$ the unit ball of $X$.

A state on $\mathcal{M}$ is a positive linear functional $\rho: \mathcal{M} \rightarrow \mathbb{C}$ of norm one. For a normal (i.e. belonging to $\mathcal{M}_{*}$ ) state $\rho$, its support, denoted by $\mathrm{s}(\rho)$, is defined as the smallest projection $p$ in $\mathcal{M}$ such that $\rho(p)=\rho(\mathbb{1})$.

There is a decomposition

$$
\mathcal{M}^{*}=\mathcal{M}_{*} \oplus \mathcal{M}_{*}^{\perp},
$$

yielding that each bounded linear functional $\varphi$ on $\mathcal{M}$ is uniquely decomposed as

$$
\varphi=\varphi_{n}+\varphi_{s}, \quad \varphi_{n} \in \mathcal{M}_{*}, \varphi_{s} \in \mathcal{M}_{*}^{\perp}
$$

into its normal and singular parts. Accordingly, there are projections $R$ and $R^{\perp}$ of norm one from $\mathcal{M}^{*}$ onto $\mathcal{M}_{*}$ and $\mathcal{M}_{*}^{\perp}$ respectively, so that

$$
R \varphi=\varphi_{n}, \quad R^{\perp} \varphi=\varphi_{s} .
$$

Moreover, $R$ and $R^{\perp}$ send the positive functionals to positive ones.

Let $(G,+)$ be an abelian semigroup. By a Markov semigroup $\left(T_{t}: t \in\right.$ $G$ ) we shall mean a semigroup of positive linear maps on $\mathcal{M}_{*}$ sending states to states. In particular, we have $T_{s+t}=T_{s} T_{t}$ for all $s, t \in G$. Considering the adjoint maps $T_{t}^{*}: \mathcal{M} \rightarrow \mathcal{M}$, we see that $\left(T_{t}^{*}: t \in G\right)$ is a semigroup of normal positive linear unital operators on $\mathcal{M}$, i.e. a quantum dynamical semigroup. We want to investigate various ergodic properties of $\left(T_{t}\right)$. The investigations of this type have been made almost exclusively for the semigroups $G=\mathbb{R}_{+}=[0, \infty)$ or $G=\mathbb{Z}_{+}=\mathbb{N} \cup\{0\}$, in the first case with the additional assumption of the continuity of the action $\mathbb{R}_{+} \ni t \mapsto T_{t}$, and in both cases with the additional assumption of complete, or at least strong, positivity of the $T_{t}^{*}$. By strong positivity 
(also called $1 \frac{1}{2}$-positivity or the Schwarz property) for a bounded unital operator $S$ on $\mathcal{M}$ is meant that $S\left(x^{*} x\right) \geqslant S(x)^{*} S(x)$ for all $x \in \mathcal{M}$ (see e.g. $[\mathbf{9}, \mathbf{1 4}])$. Our approach unifies the two cases, and includes also the case of multiparameter semigroups. Moreover, it turns out that the continuity assumption is very often redundant, as well as the assumption of stronger positivity of the $T_{t}^{*}$.

For a Markov semigroup $\left(T_{t}: t \in G\right)$, we denote by Fix $T$ its fixedpoint space, i.e.

$$
\text { Fix } T=\left\{\varphi \in \mathcal{M}_{*}: T_{t} \varphi=\varphi \text { for all } t \in G\right\},
$$

and in line with our notational convention

$$
(\text { Fix } T)^{h}=\left\{\varphi \in \mathcal{M}_{*}^{h}: T_{t} \varphi=\varphi \text { for all } t \in G\right\} .
$$

Accordingly, by Fix $T^{*}$ we denote the fixed-point space of the semigroup $\left(T_{t}^{*}: t \in G\right)$, i.e.

$$
\text { Fix } T^{*}=\left\{x \in \mathcal{M}: T_{t}^{*} x=x \text { for all } t \in G\right\},
$$

and

$$
\left(\operatorname{Fix} T^{*}\right)^{h}=\left\{x \in \mathcal{M}^{h}: T_{t}^{*} x=x \text { for all } t \in G\right\} .
$$

Observe that always $0, \mathbb{1} \in \operatorname{Fix} T^{*}$, while it may happen that $\operatorname{Fix} T=\{0\}$.

\section{Semigroups: limits and means. Generalised averages}

Much of what will be said below belongs to the folklore of semigroup theory. However, for the convenience of the reader and since it will be referred to repeatedly in the sequel, we present all the needed material here.

Since an important role in many considerations for the semigroups $\mathbb{R}_{+}$ or $\mathbb{Z}_{+}$is played by limits at infinity such as $\lim _{t \rightarrow \infty} T_{t}$ or $\lim _{n \rightarrow \infty} T^{n}$, our first step consists in generalising the notion of limit to an arbitrary abelian semigroup $G$. To this end, suppose that the following relation ' $\leqslant$ ' between elements of $G$ is given making $(G, \leqslant)$ a directed set:

$$
s \leqslant t \text { if } s=t \text { or there is } u \in G \text { such that } t=s+u \text {. }
$$

It is obvious that the natural orders on $\mathbb{Z}_{+}, \mathbb{R}_{+}$, or the multiparameter semigroup $\mathbb{R}_{+}^{d}=\left\{\left(t_{1}, \ldots, t_{d}\right): t_{i} \geqslant 0, i=1, \ldots, d\right\}$ are defined by (1). The relation ' $\leqslant$ ' defined by condition (1) was considered in $[\mathbf{5}$, Section 1.3.9], and without the requirement $s=t$ in [19, p. 75]. The notion ' $\lim _{t \in G}$ ', which shall be crucial in our further considerations, was (in the language of filters) exploited in [7] for an abelian semigroup of operators acting on a Banach space. Quite recently, this notion has also been exploited in the same setup in $[\mathbf{1 1}]$. 
Let $\left\{a_{t}: t \in G\right\}$ be a (bounded) net of real numbers. We define $\limsup a_{t}$ by the formula $t \in G$

$$
\limsup _{t \in G} a_{t}=\inf _{s \in G} \sup \left\{a_{t}: t \geqslant s\right\} .
$$

(As usual, we write $t \geqslant s$ if $s \leqslant t$.) It is seen that for a real number $\alpha$ we have $\limsup _{t \in G} a_{t} \leqslant \alpha$ if and only if for any $\varepsilon>0$ there is $t_{0} \in G$ such that for all $t \geqslant t_{0}$ the inequality $a_{t} \leqslant \alpha+\varepsilon$ holds.

Let $\ell^{\infty}(G)$ be the Banach space of all bounded functions on $G$. A functional $m \in \ell^{\infty}(G)^{*}$ is called a mean if it satisfies

(i) $m(\bar{f})=\overline{m(f)}$ for all $f \in \ell^{\infty}(G)$,

(ii) $\inf _{t \in G} f(t) \leqslant m(f) \leqslant \sup _{t \in G} f(t)$ for all real-valued $f \in \ell^{\infty}(G)$.

Equivalently, $m$ is a positive functional of norm one, i.e. a state on $\ell^{\infty}(G)$. Denote by $\delta_{t}$ a functional on $\ell^{\infty}(G)$ defined as $\delta_{t}(f)=f(t)$. Then it is known that $m$ lies in the weak ${ }^{*}$-closure of the convex hull of all $\delta_{t}$ with $t \in G$. Consequently, there is a net $\left\{g_{\gamma}: \gamma \in \Gamma\right\}$ of elements from this convex hull such that

$$
m=\text { weak }^{*}-\lim _{\gamma \in \Gamma} g_{\gamma}
$$

(cf. e.g. [13]). Since each $g_{\gamma}$ has the form $g_{\gamma}=\sum_{i=1}^{n_{\gamma}} \lambda_{i}^{\gamma} \delta_{t_{i}^{\gamma}}$, with $n_{\gamma}$ positive integers, $\lambda_{i}^{\gamma}$, for $i=1, \ldots, n_{\gamma}$, positive numbers satisfying $\sum_{i=1}^{n_{\gamma}} \lambda_{i}^{\gamma}=1$, and $t_{i}^{\gamma}$, for $i=1, \ldots, n_{\gamma}$, elements from $G$, we obtain

$$
m=\text { weak }^{*}-\lim _{\gamma} \sum_{i=1}^{n_{\gamma}} \lambda_{i}^{\gamma} \delta_{t_{i}^{\gamma}},
$$

with the nets $\left\{n_{\gamma}: \gamma \in \Gamma\right\},\left\{\lambda_{i}^{\gamma}: \gamma \in \Gamma\right\}$, and $\left\{t_{i}^{\gamma}: \gamma \in \Gamma\right\}$ as above.

For $s \in G$ and $f \in \ell^{\infty}(G)$, define the function $f_{s} \in \ell^{\infty}(G)$ by the formula

$$
f_{s}(t)=f(t+s), \quad t \in G .
$$

If the mean $m$ satisfies

$$
m\left(f_{s}\right)=m(f) \text { for all } f \in \ell^{\infty}(G) \text { and all } s \in G,
$$

then it is called an invariant mean. It is known that such means exist on $\ell^{\infty}(G)$ for any abelian semigroup $G$ (see e.g. [2] or [13]). 
Note that, if for $f, g \in \ell^{\infty}(G)$ we have $f(t)=g(t)$ for all $t \geqslant t_{0}$, then $m(f)=m(g)$ for each invariant mean $m$ (it follows from the invariance of $m$ and the equality $f_{t_{0}}=g_{t_{0}}$ ). For such a mean we also have

$$
m(f)=0
$$

if $\lim _{t \in G} f(t)=0$. Indeed, for any $\varepsilon>0$ there is $t_{0}$ such that $\left|f_{t_{0}}\right|<\varepsilon$, hence

$$
|m(f)|=\left|m\left(f_{t_{0}}\right)\right| \leqslant \varepsilon .
$$

It follows that if there exists $\lim _{t \in G} f(t)=c$, then

$$
m(f)=c=\lim _{t \in G} f(t) .
$$

A generalised average on $G$ is a net $\left\{A_{\gamma}: \gamma \in \Gamma\right\}$ of states on $\ell^{\infty}(G)$ such that, for any $f \in \ell^{\infty}(G)$ and any $t \in G$,

$$
\lim _{\gamma \in \Gamma}\left|A_{\gamma}\left(f_{t}\right)-A_{\gamma}(f)\right|=0 .
$$

Fundamental examples of such averages are the following Cesàro means:

$-G=\mathbb{Z}_{+}, \Gamma=\{1,2, \ldots\}$,

$$
A_{n}(f)=\frac{1}{n} \sum_{k=0}^{n-1} f(k), \quad f=(f(k))_{k \in \mathbb{Z}_{+}},
$$

- $G=\mathbb{R}_{+}, \Gamma=(0, \infty)$. Here the means $A_{t}$ are defined on the space $\mathcal{C}_{b}(G)$ of bounded continuous functions on $G$, where $G$ is endowed with the natural topology,

$$
A_{t}(f)=\frac{1}{t} \int_{0}^{t} f(s) d s,
$$

the following Borel means:

- $G=\mathbb{Z}_{+}, \Gamma=[0, \infty)$,

$$
A_{\gamma}(f)=e^{-\gamma} \sum_{k=0}^{\infty} \frac{\gamma^{k}}{k !} f(k), \quad f=(f(k))_{k \in \mathbb{Z}_{+}},
$$

and the following Abel means:

$-G=\mathbb{Z}_{+}, \Gamma=(1, \infty)$,

$$
A_{\gamma}(f)=(\gamma-1) \sum_{k=0}^{\infty} \frac{f(k)}{\gamma^{k+1}}, \quad f=(f(k))_{k \in \mathbb{Z}_{+}},
$$

where in the net $\left\{A_{\gamma}: \gamma \in(1, \infty)\right\}$ the set $(1, \infty)$ is directed according to the order reversed to the natural one. 
An interesting example of generalised averages for arbitrary abelian semigroups is given by the following notion due to Hugenholtz (see [16]). A net $\left\{h_{\gamma}: \gamma \in \Gamma\right\}$ in $\ell^{\infty}(G)$ is called an $M$-net (not to be confused with M-nets considered in [6]) if

(i) for each $\gamma \in \Gamma$, we have $h_{\gamma} \geqslant 0$,

(ii) for each $\gamma \in \Gamma$, we have $\sum_{t \in G} h_{\gamma}(t)=1$,

(iii) for each $s \in G$, we have

$$
\lim _{\gamma \in \Gamma} \sum_{t \in G}\left|h_{\gamma}(t+s)-h_{\gamma}(t)\right|=0 .
$$

The lemma below shows how M-nets can be employed for the construction of generalised averages.

Lemma 1. Assume that the order $\leqslant$ on $G$ enjoys the property $s \leqslant t$ if and only if there is $u \in G$ such that $t=s+u$, and put

$$
A_{\gamma}(f)=\sum_{t \in G} h_{\gamma}(t) f(t), \quad f \in \ell^{\infty}(G),
$$

where $\left\{h_{\gamma}: \gamma \in \Gamma\right\}$ is an M-net. Then $\left\{A_{\gamma}: \gamma \in \Gamma\right\}$ is a generalised average on $G$.

Proof: We have

$$
\sum_{t \ngtr s} h_{\gamma}(t)=\sum_{t \in G} h_{\gamma}(t)-\sum_{t \geqslant s} h_{\gamma}(t)=\sum_{t \in G} h_{\gamma}(t)-\sum_{t \in G} h_{\gamma}(t+s),
$$

which yields

$$
\sum_{t \ngtr s} h_{\gamma}(t) \leqslant \sum_{t \in G}\left|h_{\gamma}(t)-h_{\gamma}(t+s)\right| .
$$

Thus, on account of condition (iii), we get

$$
\lim _{\gamma \in \Gamma} \sum_{t \ngtr s} h_{\gamma}(t)=0 .
$$

From this we obtain for each $f \in \ell^{\infty}(G)$,

$$
\lim _{\gamma \in \Gamma} \sum_{t \ngtr s} h_{\gamma}(t) f(t)=0 .
$$


Now, we have for each $s \in G$,

$$
\sum_{t \geqslant s} h_{\gamma}(t) f(t)=\sum_{t \in G} h_{\gamma}(t+s) f(t+s),
$$

and thus

$$
\begin{aligned}
& \left|A_{\gamma}\left(f_{s}\right)-A_{\gamma}(f)\right|=\left|\sum_{t \in G} h_{\gamma}(t) f_{s}(t)-\sum_{t \in G} h_{\gamma}(t) f(t)\right| \\
& =\left|\sum_{t \in G} h_{\gamma}(t) f(t+s)-\sum_{t \in G} h_{\gamma}(t) f(t)\right| \\
& \quad \leqslant\left|\sum_{t \in G} h_{\gamma}(t) f(t+s)-\sum_{t \geqslant s} h_{\gamma}(t) f(t)\right|+\left|\sum_{t \ngtr s} h_{\gamma}(t) f(t)\right| \\
& =\left|\sum_{t \in G} h_{\gamma}(t) f(t+s)-\sum_{t \in G} h_{\gamma}(t+s) f(t+s)\right|+\left|\sum_{t \ngtr s} h_{\gamma}(t) f(t)\right| \\
& \quad \leqslant \sum_{t \in G}\left|h_{\gamma}(t)-h_{\gamma}(t+s)\right||f(t+s)|+\left|\sum_{t \ngtr s} h_{\gamma}(t) f(t)\right| .
\end{aligned}
$$

The first sum on the right hand side tends to 0 by virtue of the definition of an M-net, and so does the second by virtue of formula (6). This shows that $\left\{A_{\gamma}: \gamma \in \Gamma\right\}$ is a generalised average on $G$.

It is clear that for an invariant mean $m$ we can define a generalised average taking e.g. $A_{\gamma}=m$ for all $\gamma$, or

$$
A_{\gamma}=\sum_{i=1}^{n_{\gamma}} \lambda_{i}^{\gamma} \delta_{t_{i}^{\gamma}}
$$

where the sum on the right hand side is as in condition (2), i.e. these sums converge to $m$. An important fact about generalised averages is that the converse also holds true, i.e. they define invariant means on $\ell^{\infty}(G)$. Namely, let $\left\{A_{\gamma}: \gamma \in \Gamma\right\}$ be a generalised average on $G$. Put

$$
\mathbb{K}=\left\{f \in \ell^{\infty}(G): \lim _{\gamma \in \Gamma} A_{\gamma}(f) \text { exists }\right\}
$$

$\mathbb{K}$ is a closed subspace of $\ell^{\infty}(G)$. Define $m_{0}: \mathbb{K} \rightarrow \mathbb{C}$ by

$$
m_{0}(f)=\lim _{\gamma \in \Gamma} A_{\gamma}(f), \quad f \in \mathbb{K} .
$$

$m_{0}$ is a linear functional of norm one on $\mathbb{K}$. By the Hahn-Banach Theorem, $m_{0}$ is extended to a linear functional $m$ of norm one on $\ell^{\infty}(G)$ 
such that $m(\mathbb{1})=1$. It follows that $m$ is a state. For any $s \in G$, we have from the definition of generalised averages the relation

$$
\lim _{\gamma \in \Gamma} A_{\gamma}\left(f_{s}-f\right)=0,
$$

and thus $f_{s}-f \in \mathbb{K}$. Consequently,

$$
0=\lim _{\gamma \in \Gamma} A_{\gamma}\left(f_{s}-f\right)=m_{0}\left(f_{s}-f\right)=m\left(f_{s}-f\right),
$$

showing that $m$ is an invariant mean. Each such mean is said to be associated with the generalised average $\left\{A_{\gamma}: \gamma \in \Gamma\right\}$.

Remark 1. For the semigroup $\mathbb{Z}_{+}$and M-nets $\left\{h_{n}^{(1)}: n=0,1, \ldots\right\}$, $\left\{h_{\gamma}^{(2)}: \gamma>0\right\}$, and $\left\{h_{\gamma}^{(3)}: \gamma>1\right\}$ defined as

$$
\begin{aligned}
& h_{n}^{(1)}(k)= \begin{cases}\frac{1}{n} & \text { for } 0 \leqslant k \leqslant n-1, \\
0 & \text { for } k \geqslant n,\end{cases} \\
& h_{\gamma}^{(2)}(k)=e^{-\gamma} \frac{\gamma^{k}}{k !}, \quad k=0,1, \ldots,
\end{aligned}
$$

and

$$
h_{\gamma}^{(3)}(k)=\frac{\gamma-1}{\gamma^{k+1}}, \quad k=0,1, \ldots,
$$

the generalised averages have the form

$$
\begin{aligned}
& A_{n}^{(1)}(f)=\frac{1}{n} \sum_{k=0}^{n-1} f(k), \\
& A_{\gamma}^{(2)}(f)=e^{-\gamma} \sum_{k=0}^{\infty} \frac{\gamma^{k}}{k !} f(k),
\end{aligned}
$$

and

$$
A_{\gamma}^{(3)}(f)=(\gamma-1) \sum_{k=0}^{\infty} \frac{f(k)}{\gamma^{k+1}},
$$

for $f \in \ell^{\infty}\left(\mathbb{Z}_{+}\right)$. Thus, we obtain the Cesàro means $A_{n}^{(1)}$, the Borel means $A_{\gamma}^{(2)}$, and the Abel means $A_{\gamma}^{(3)}$. (See also [26] where M-nets were used in deriving some ergodic theorems on von Neumann algebras.)

A central role in our considerations will be played by the following construction. Let $M$ be a bounded linear functional on $\ell^{\infty}(G)$. Let $\widehat{\varphi}=$ 
$\left(\varphi_{t}\right)_{t \in G} \in \ell^{\infty}\left(G, \mathcal{M}^{*}\right)$ - the bounded functions on $G$ with values in $\mathcal{M}^{*}$. For such a map $\widehat{\varphi}$, we define a functional $\widetilde{M}_{\widehat{\varphi}} \in \mathcal{M}^{*}$ by

$$
\widetilde{M}_{\widehat{\varphi}}(x)=M\left(\varepsilon_{x}(\widehat{\varphi})\right), \quad x \in \mathcal{M},
$$

where $\varepsilon_{x}(\widehat{\varphi}) \in \ell^{\infty}(G)$ is defined by

$$
\varepsilon_{x}(\widehat{\varphi})(t)=\varphi_{t}(x), \quad t \in G .
$$

It is easily seen that if $M$ is a state and all $\varphi_{t}$ are states, then $\widetilde{M}_{\widehat{\varphi}}$ is a state too. In what follows, we shall usually employ the notation $\widetilde{M}_{\left[\varphi_{t}\right]}$ instead of $\widetilde{M}_{\widehat{\varphi}}$ in such cases.

If $\left(T_{t}: t \in G\right)$ is a Markov semigroup, then $\left(T_{t}^{* *}: t \in G\right)$ is clearly a semigroup of positive linear maps on $\mathcal{M}^{*}$. We define a map $M_{T}: \mathcal{M}^{*} \rightarrow$ $\mathcal{M}^{*}$ by the formula

$$
M_{T}(\varphi)=\widetilde{M}_{\left[T_{t}^{* *} \varphi\right]}, \quad \varphi \in \mathcal{M}^{*},
$$

where $\left[T_{t}^{* *} \varphi\right]$ is shorthand for the map $t \mapsto T_{t}^{* *} \varphi$ from $\ell^{\infty}\left(G, \mathcal{M}^{*}\right)$.

The above construction will be employed in the following two situations.

1. $M$ is an invariant mean $m$.

Then from formula (2) we obtain

$$
\widetilde{m}_{\left[\varphi_{t}\right]}(x)=m\left(\left[t \mapsto \varphi_{t}(x)\right]\right)=\lim _{\gamma} \sum_{i=1}^{n_{\gamma}} \lambda_{i}^{\gamma} \varphi_{t_{i}^{\gamma}}(x),
$$

which means that

$$
\widetilde{m}_{\left[\varphi_{t}\right]}=\text { weak }^{*}-\lim _{\gamma} \varphi_{\gamma}
$$

where

$$
\varphi_{\gamma}=\sum_{i=1}^{n_{\gamma}} \lambda_{i}^{\gamma} \varphi_{t_{i}^{\gamma}} .
$$

As before, we see that if $\varphi_{t}=\psi_{t}$ for all $t \geqslant t_{0}$, then $\widetilde{m}_{\left[\varphi_{t}\right]}=\widetilde{m}_{\left[\psi_{t}\right]}$.

For a Markov semigroup $\left(T_{t}: t \in G\right), \varphi \in \mathcal{M}^{*}$, and arbitrary $x \in \mathcal{N}$, define $f^{x} \in \ell^{\infty}(G)$ by

$$
f^{x}(t)=\left(T_{t}^{* *} \varphi\right)(x), \quad t \in G .
$$

Then for any $s \in G$, we have

$$
f^{T_{s}^{*} x}(t)=\left(T_{t}^{* *} \varphi\right)\left(T_{s}^{*} x\right)=\left(T_{t+s}^{* *} \varphi\right)(x)=\left(f^{x}\right)_{s}(t),
$$

and thus

$$
\begin{aligned}
\left(T_{s}^{* *} m_{T}(\varphi)\right)(x) & =m_{T}(\varphi)\left(T_{s}^{*} x\right)=m\left(f^{T_{s}^{*} x}\right) \\
& =m\left(\left(f^{x}\right)_{h}\right)=m\left(f^{x}\right)=m_{T}(\varphi)(x),
\end{aligned}
$$


which means that $m_{T}(\varphi)$ is $\left(T_{t}^{* *}\right)$-invariant. Moreover, $m_{T}$ is a positive projection of norm one onto the space of all $\left(T_{t}^{* *}\right)$-fixed points of $\mathcal{M}^{*}$ sending states to states (the map $m_{T}$ was employed in [25]).

2. $M=A_{\gamma}$ - one single term of a generalised average.

Then

$$
\left(A_{\gamma}\right)_{T}(\varphi)=\left(\widetilde{A_{\gamma}}\right)_{\left[T_{t}^{* *} \varphi\right]}, \quad \varphi \in \mathcal{M}^{*} .
$$

In particular, if $\varphi \in \operatorname{Fix} T$, then $T_{t} \varphi=\varphi$, hence

$$
\left(A_{\gamma}\right)_{T}(\varphi)=\varphi
$$

Let now $\widetilde{\varphi}$ be an arbitrary weak ${ }^{*}$-limit point of the net $\left\{\left(A_{\gamma}\right)_{T}(\varphi)\right\}$, where $\varphi \in \mathcal{M}^{*}$. Then there is a subnet $\left\{\gamma^{\prime}\right\}$ such that for each $x \in \mathcal{M}$

$$
\lim _{\gamma^{\prime}}\left(A_{\gamma^{\prime}}\right)_{T}(\varphi)(x)=\widetilde{\varphi}(x) .
$$

Let $m$ be an invariant mean associated with the net $\left\{A_{\gamma^{\prime}}\right\}$. Then for each fixed $x \in \mathcal{M}$, we have

$$
\left(A_{\gamma^{\prime}}\right)_{T}(\varphi)(x)=\left(\widetilde{A_{\gamma^{\prime}}}\right)_{\left[T_{t}^{* *} \varphi\right]}(x)=A_{\gamma^{\prime}}\left(\left[t \mapsto\left(T_{t}^{* *} \varphi\right)(x)\right]\right),
$$

and passing to the limit, we obtain

$$
\begin{aligned}
\widetilde{\varphi}(x) & =\lim _{\gamma^{\prime}}\left(A_{\gamma^{\prime}}\right)_{T}(\varphi)(x)=\lim _{\gamma^{\prime}} A_{\gamma^{\prime}}\left(\left[t \mapsto\left(T_{t}^{* *} \varphi\right)(x)\right]\right) \\
& =m\left(\left[t \mapsto\left(T_{t}^{* *} \varphi\right)(x)\right]\right)=\widetilde{m}_{\left[T_{t}^{* *} \varphi\right]}(x)=m_{T}(\varphi)(x),
\end{aligned}
$$

showing that $\tilde{\varphi}$ is $\left(T_{t}^{* *}\right)$-invariant.

It is interesting to compare a generalised average $\left\{\left(A_{\gamma}\right)_{T}: \gamma \in \Gamma\right\}$ with the notion of a weak $\left(T_{t}^{* *}\right)$-ergodic net considered e.g. in [19, Chapter 2]. Such a net $\left\{\widehat{A}_{\gamma}: \gamma \in \Gamma\right\}$ is defined in our setting by the following conditions:

(E1) each $\widehat{A}_{\gamma}$ is a linear operator on $\mathcal{M}^{*}$,

(E2) for each $\varphi \in \mathcal{M}^{*}$ and all $\gamma \in \Gamma$, we have $\widehat{A}_{\gamma} \varphi \in \overline{\operatorname{conv}\left\{T_{t}^{* *} \varphi: t \in G\right\}}$, where the closure is in the weak (equivalently, norm) topology,

(E3) the $\widehat{A}_{\gamma}$ are equicontinuous,

(E4) for every $\varphi \in \mathcal{M}^{*}$ and every $s \in G$,

$$
\text { weak- } \lim _{\gamma}\left(\widehat{A}_{\gamma} T_{s}^{* *} \varphi-\widehat{A}_{\gamma} \varphi\right)=0 \text {. }
$$

Since the elements $\left(A_{\gamma}\right)_{T}$ of a generalised average are positive linear maps of norm one on $\mathcal{M}^{*}$, conditions (E1) and (E3) above are satisfied. 
As for condition (E4), we have for arbitrary $x \in \mathcal{M}$, every $\varphi \in \mathcal{M}^{*}$, and every $s \in G$,

$$
\left(A_{\gamma}\right)_{T}\left(T_{s}^{* *} \varphi\right)(x)=A_{\gamma}\left(\left[t \mapsto\left(T_{t+s} \varphi\right)(x)\right]\right)=A_{\gamma}\left(f_{s}^{x}\right),
$$

and

$$
\left(A_{\gamma}\right)_{T}(\varphi)(x)=A_{\gamma}\left(\left[t \mapsto\left(T_{t} \varphi\right)(x)\right]\right)=A_{\gamma}\left(f^{x}\right)
$$

and thus on account of condition (5) for generalised averages we get

$$
\left|\left(\left(A_{\gamma}\right)_{T}\left(T_{s}^{* *} \varphi\right)-\left(A_{\gamma}\right)_{T}(\varphi)\right)(x)\right|=\left|A_{\gamma}\left(f_{s}^{x}\right)-A_{\gamma}\left(f^{x}\right)\right| \underset{\gamma}{\longrightarrow} 0,
$$

so condition (E4) is satisfied if instead of a weak limit we take a weak* limit. A similar situation occurs with condition (E2). Since $A_{\gamma}$ are states on $\ell^{\infty}(G)$, they belong to the weak ${ }^{*}$-closure of $\operatorname{conv}\left\{\delta_{t}: t \in G\right\}$ which yields that $\left(A_{\gamma}\right)_{T}(\varphi)$ belong to the weak ${ }^{*}$-closure of $\operatorname{conv}\left\{T_{t}^{* *} \varphi: t \in\right.$ $G\}$ instead of the weak-closure. Consequently, the generalised average $\left\{\left(A_{\gamma}\right)_{T}: \gamma \in \Gamma\right\}$ could be named a weak* $\left(T_{t}^{* *}\right)$-ergodic net. However, it is an open problem if for such nets results analogous to e.g. Theorem II.1.5 or Theorem II.1.9 in [19] hold.

Remark 2. Let $\left\{A_{\gamma}: \gamma \in \Gamma\right\}$ be an arbitrary generalised average on $G$. Since the $A_{\gamma}$ are, in principle, arbitrary states on $\ell^{\infty}(G)$ (the crucial property (5) refers to the limiting behaviour of $A_{\gamma}(f)$ ), we cannot expect that $\left(A_{\gamma}\right)_{T}(\varphi) \in \mathcal{M}_{*}$ for $\varphi \in \mathcal{M}_{*}$. However, if the $A_{\gamma}$ are defined by means of M-nets as in Lemma 1 , then this condition is satisfied. Indeed, in this case we have

$$
\left(A_{\gamma}\right)_{T}(\varphi)=\sum_{t \in G} h_{\gamma}(t) T_{t} \varphi
$$

and the series on the right hand side converges in norm since

$$
\sum_{t \in G}\left\|h_{\gamma}(t) T_{t} \varphi\right\|=\sum_{t \in G} h_{\gamma}(t)\left\|T_{t} \varphi\right\| \leqslant \sum_{t \in G} h_{\gamma}(t)\|\varphi\|=\|\varphi\|,
$$

by properties (i) and (ii) of M-nets. Consequently, if $\varphi \in \mathcal{M}_{*}$, then $h_{\gamma}(t) T_{t} \varphi \in \mathcal{M}_{*}$, and the norm-convergence implies $\left(A_{\gamma}\right)_{T}(\varphi) \in \mathcal{M}_{*}$. Observe that in this case the generalised average $\left\{\left(A_{\gamma}\right)_{T}: \gamma \in \Gamma\right\}$ is exactly a weak $\left(T_{t}\right)$-ergodic net as in [19, Chapter 2].

Let $\left(T_{t}: t \in G\right)$ be a Markov semigroup, and let $\varphi \in \mathcal{M}_{*}$. We are interested in the behaviour of the orbit $\left(T_{t} \varphi: t \in G\right)$. In particular, when this orbit is close to some set. Thus, let $\mathcal{A} \subset \mathcal{M}_{*}$ and let $\alpha \geqslant 0$. Following the idea in $[\mathbf{2 1}]$ and $[\mathbf{2 2}], \mathcal{A}$ is said to be an $\alpha$-constrictor for the semigroup $\left(T_{t}\right)$ if for each normal state $\varphi$ we have

$$
\limsup _{t \in G} \operatorname{dist}\left(T_{t} \varphi, \mathcal{A}\right) \leqslant \alpha
$$


where $\operatorname{dist}(\varphi, \mathcal{A})$ denotes the distance of the element $\varphi$ from the set $\mathcal{A}$, i.e.

$$
\operatorname{dist}(\varphi, \mathcal{A})=\inf \{\|\varphi-\psi\|: \psi \in \mathcal{A}\}
$$

(Note that this definition of an $\alpha$-constrictor is slightly different, and less demanding, than that in [9] where condition (8) is assumed to hold for any hermitian $\varphi$ in the unit ball.) It is easily seen that $\mathcal{A}$ is an $\alpha$ constrictor for $\left(T_{t}\right)$ if and only if for every normal state $\varphi$ there is $\psi_{t} \in \mathcal{A}$ such that

$$
\limsup _{t \in G}\left\|T_{t} \varphi-\psi_{t}\right\| \leqslant \alpha
$$

i.e. for every $\varphi \in \mathcal{M}_{*}^{+}$, there are $\psi_{t} \in \mathcal{A}$ such that

$$
\limsup _{t \in G}\left\|T_{t} \varphi-\left(\|\varphi\| \psi_{t}\right)\right\| \leqslant \alpha\|\varphi\|,
$$

which in turn is equivalent to the condition: for any $\varphi \in \mathcal{M}_{*}^{+}$and any $\beta>$ $\alpha$, there are $\psi_{t} \in \mathcal{A}$ and $t_{0} \in G$ such that for all $t \geqslant t_{0}$

$$
\left\|T_{t} \varphi-\left(\|\varphi\| \psi_{t}\right)\right\| \leqslant \beta\|\varphi\| \text {. }
$$

In particular, for $\alpha=0$, we simply have for every normal state $\varphi$

$$
\lim _{t \in G}\left\|T_{t} \varphi-\psi_{t}\right\|=0
$$

i.e. for every $\varphi \in \mathcal{M}_{*}^{+}$,

$$
\lim _{t \in G}\left\|T_{t} \varphi-\left(\|\varphi\| \psi_{t}\right)\right\|=0 .
$$

The last condition leads in a natural way to the following definition. $\mathcal{A}$ is said to be a weak 0 -constrictor (i.e. $\sigma\left(\mathcal{M}_{*}, \mathcal{M}\right) 0$-constrictor) for the semigroup $\left(T_{t}\right)$ if for every $\varphi \in \mathcal{M}_{*}^{+}$, there are $\psi_{t} \in \mathcal{A}$ such that

$$
\text { weak- } \lim _{t \in G}\left(T_{t} \varphi-\|\varphi\| \psi_{t}\right)=0 \text {. }
$$

Remark 3. The notion of constrictor, also called attractor, was considered in a number of papers. In particular, in [7] quasi-constrictivity of a semigroup of operators acting on a Banach space was introduced, while in [18] constrictive operators on $L^{1}$ were studied. This type of investigation together with constrictive operators on $C(X)$ was performed also in [31]. 


\section{Mean ergodicity}

Consider now the notion of mean ergodicity. For the semigroups $\left(T_{t}\right.$ : $\left.t \in \mathbb{R}_{+}\right)$or $\left(T^{n}: n \in \mathbb{Z}_{+}\right)$, it is usually defined as the existence of norm limits of the Cesàro means. Namely, for each $\varphi \in \mathcal{M}_{*}$, the limits

$$
\lim _{t \rightarrow \infty} \frac{1}{t} \int_{0}^{t} T_{s} \varphi d s=m_{T}(\varphi) \quad \text { or } \quad \lim _{n \rightarrow \infty} \frac{1}{n} \sum_{k=0}^{n-1} T^{k} \varphi=m_{T}(\varphi)
$$

exist. Since the $m_{T}$ are examples of invariant means, a natural definition of ergodicity for the semigroup $\left(T_{t}: t \in G\right)$ could be as follows. $\left(T_{t}\right)$ is said to be mean ergodic if for every $\varphi \in \mathcal{M}_{*}$ we have $m_{T}(\varphi) \in \mathcal{M}_{*}$, where $m$ is an invariant mean on $\ell^{\infty}(G)$. Below, we show that this property does not depend on the choice of mean $m$. Notice that the seemingly abstract definition above gives, in the cases of concrete semigroups $\mathbb{R}_{+}$or $\mathbb{Z}_{+}$ exactly the customary notion of mean ergodicity. Namely, on account of formula (7), we have for every $\varphi \in \mathcal{M}_{*}$

$$
m_{T}(\varphi)=\text { weak- } \lim _{\gamma} \sum_{i=1}^{n_{\gamma}} \lambda_{i}^{\gamma} T_{t_{i}^{\gamma}} \varphi,
$$

and the condition $m_{T}(\varphi) \in \mathcal{M}_{*}$ means that $m_{T}(\varphi) \in \overline{\operatorname{conv}\left\{T_{t} \varphi: t \in G\right\}}$, where the closure is in the $\sigma\left(\mathcal{M}_{*}, \mathcal{M}\right)$ - (i.e. weak) topology. From Mazur's Theorem, it follows that $m_{T}(\varphi)$ belongs to the closure of $\operatorname{conv}\left\{T_{t} \varphi: t \in\right.$ $G\}$ in the norm topology. Since $m_{T}(\varphi)$ is obviously $\left(T_{t}\right)$-invariant, the mean ergodic theorem ([19, Theorem II.1.1]) yields that $m_{T}(\varphi)$ is a limit of the Cesàro means.

It is interesting to compare the definition of mean ergodicity given above with the one formulated for a general semigroup of mappings in [30]. We shall do this after Remark 4 when we have some additional notions at our disposal.

Lemma 2. Let $\varphi \in \mathcal{M}_{*}$. If $m_{T}^{\prime}(\varphi) \in \mathcal{M}_{*}$ for some invariant mean $m^{\prime}$, then for every invariant mean $m$ we have $m_{T}(\varphi)=m_{T}^{\prime}(\varphi)$.

Proof: As seen before, $m_{T}^{\prime}(\varphi)$ belongs to the closure of $\operatorname{conv}\left\{T_{t} \varphi: t \in G\right\}$ in the norm topology, so there is a net $\left\{\varphi_{\gamma}\right\} \subset \operatorname{conv}\left\{T_{t} \varphi: t \in G\right\}$ such that

$$
m_{T}^{\prime}(\varphi)=\text { norm- } \lim _{\gamma} \varphi_{\gamma} .
$$

Take an arbitrary $\varepsilon>0$, and let $\gamma_{0}$ be such that

$$
\left\|m_{T}^{\prime}(\varphi)-\varphi_{\gamma_{0}}\right\|<\varepsilon .
$$


Since

$$
\varphi_{\gamma_{0}}=\sum_{i=1}^{r} \lambda_{i} T_{t_{i}} \varphi
$$

for some $\lambda_{i} \geqslant 0, \sum_{i=1}^{r} \lambda_{i}=1$, and $t_{i} \in G$, we have

$$
m_{T}\left(\varphi_{\gamma_{0}}\right)=\sum_{i=1}^{r} \lambda_{i} m_{T}\left(T_{t_{i}} \varphi\right)=m_{T}(\varphi)
$$

and

$$
m_{T}\left(m_{T}^{\prime}(\varphi)\right)=m_{T}^{\prime}(\varphi)
$$

because $m_{T}^{\prime}(\varphi) \in \operatorname{Fix} T$. Consequently,

$$
\varepsilon>\left\|m_{T}\left(m_{T}^{\prime}(\varphi)-\varphi_{\gamma_{0}}\right)\right\|=\left\|m_{T}^{\prime}(\varphi)-m_{T}(\varphi)\right\|,
$$

showing the claim.

In [24], under the assumption $\operatorname{Fix} T \neq\{0\}$, a positive normal normone projection $\mathbb{E}: \mathcal{M} \rightarrow$ Fix $T^{*}$ was defined by the formula

$$
\mathbb{E}=\left(R \circ m_{T} \mid \mathcal{M}_{*}\right)^{*},
$$

(observe that $R \circ m_{T} \mid \mathcal{M}_{*}$ is a map from $\mathcal{M}_{*}$ into $\mathcal{M}_{*}$, so its adjoint maps $\mathcal{M}$ into $\mathcal{M})$. It was shown there that the following assertions hold:

(i) $\operatorname{Fix} T=\left\{\varphi \circ \mathbb{E}: \varphi \in \mathcal{M}_{*}\right\}$,

(ii) $T_{t}^{*} \mathbb{E}=\mathbb{E} T_{t}^{*}=\mathbb{E}$ for each $t \in G$; in particular, Range $\mathbb{E} \subset \mathrm{Fix} T^{*}$,

(cf. [24, Theorem 11]). However, even without the assumption Fix $T \neq$ $\{0\}$ a map $\mathbb{E}$ may still be defined by formula (11), and it is seen that also in this case assertions (i) and (ii) hold. Indeed, observe first that for every $\varphi \in \mathcal{M}^{*}$ and every $t \in G$, we have, by the definition of $m_{T}$,

$$
m_{T}(\varphi) \circ T_{t}^{*}=m_{T}(\varphi),
$$

and, on account of [24, Proposition 2], the following implication holds:

$$
\varphi \circ T_{t}^{*}=\varphi \quad \text { implies }(R \varphi) \circ T_{t}^{*}=R \varphi \text {. }
$$

These two facts yield

$$
\left(R \circ m_{T}\right)(\varphi) \circ T_{t}^{*}=\left(R \circ m_{T}\right)(\varphi) .
$$


Consequently, for each $\varphi \in \mathcal{M}_{*}$ we have

$$
\begin{aligned}
\varphi\left(T_{t}^{*}(\mathbb{E} x)\right) & =\varphi\left(T_{t}^{*}\left(R \circ m_{T} \mid \mathcal{M}_{*}\right)^{*}(x)\right)=\left(T_{t} \varphi\right)\left(\left(R \circ m_{T} \mid \mathcal{M}_{*}\right)^{*}(x)\right) \\
& =\left(R \circ m_{T} \mid \mathcal{M}_{*}\right)\left(T_{t} \varphi\right)(x)=\left(R \circ m_{T} \mid \mathcal{M}_{*}\right)(\varphi)(x) \\
& =\varphi\left(\left(R \circ m_{T} \mid \mathcal{M}_{*}\right)^{*}(x)\right)=\varphi(\mathbb{E} x),
\end{aligned}
$$

showing that

$$
T_{t}^{*} \mathbb{E}=\mathbb{E},
$$

and

$$
\begin{aligned}
\varphi\left(\mathbb{E}\left(T_{t}^{*} x\right)\right) & =\varphi\left(\left(R \circ m_{T} \mid \mathcal{M}_{*}\right)^{*}\left(T_{t}^{*} x\right)\right)=\left(R \circ m_{T} \mid \mathcal{M}_{*}\right)(\varphi)\left(T_{t}^{*} x\right) \\
& =\left(R \circ m_{T} \mid \mathcal{M}_{*}\right)(\varphi)(x)=\varphi\left(\left(R \circ m_{T} \mid \mathcal{M}_{*}\right)^{*} x\right)=\varphi(\mathbb{E} x),
\end{aligned}
$$

showing that

$$
\mathbb{E} T_{t}^{*}=\mathbb{E} .
$$

Now, since $\mathbb{E} T_{t}^{*}=\mathbb{E}$, we clearly have $\left\{\varphi \circ \mathbb{E}: \varphi \in \mathcal{M}_{*}\right\} \subset$ Fix $T$. On the other hand, for $\varphi \in \operatorname{Fix} T$ we have $m_{T}(\varphi)=\varphi$, and thus $\left(R \circ m_{T}\right)(\varphi)=\varphi$, which yields for each $x \in \mathcal{M}$,

$$
\varphi(\mathbb{E} x)=\varphi\left(\left(R \circ m_{T} \mid \mathcal{M}_{*}\right)^{*} x\right)=\left(R \circ m_{T}\right)(\varphi)(x)=\varphi(x),
$$

showing that $\varphi=\varphi \circ \mathbb{E}$. Hence $\operatorname{Fix} T \subset\left\{\varphi \circ \mathbb{E}: \varphi \in \mathcal{M}_{*}\right\}$. Now it is easily seen that the following equivalence holds:

$$
\text { Fix } T=\{0\} \quad \text { if and only if } \mathbb{E}=0 .
$$

In general, a (normal) projection $\mathbb{E}: \mathcal{M} \rightarrow$ Fix $T^{*}$ satisfying conditions (i) and (ii) is said to be a quasi-ergodic projection, while if $\mathbb{E}$ is defined by formula (11), then it is called the quasi-ergodic projection associated with $\mathrm{m}$. We have the following characterisation of mean ergodicity in terms of the quasi-ergodic projection.

Proposition 3. Let $\mathbb{E}$ be the quasi-ergodic projection associated with an invariant mean $m$. The following conditions are equivalent:

(i) $\left(T_{t}\right)$ is mean ergodic,

(ii) $\mathbb{E}(\mathcal{M})=\operatorname{Fix} T^{*}$,

(iii) $\mathbb{E}$ is unital.

Proof: (i) $\Longrightarrow$ (ii) The condition $m_{T}\left(\mathcal{M}_{*}\right) \subset \mathcal{M}_{*}$ yields $\mathbb{E}=\left(m_{T} \mid \mathcal{M}_{*}\right)^{*}$. For every $\varphi \in \mathcal{M}_{*}$, every $x \in \operatorname{Fix} T^{*}$, and every $t \in G$, we have

$$
\varphi(x)=\varphi\left(T_{t}^{*} x\right)=\left(T_{t} \varphi\right)(x),
$$

hence

$$
\varphi(x)=\widetilde{m}_{\left[T_{t} \varphi\right]}(x)=m_{T}(\varphi)(x)=\varphi(\mathbb{E} x) .
$$

Consequently,

$$
\mathbb{E} x=x \quad \text { for } x \in \operatorname{Fix} T^{*},
$$

showing that $E$ is surjective. 
(ii) $\Longrightarrow$ (iii) Obvious, because $\mathbb{1} \in$ Fix $T^{*}$.

(iii) $\Longrightarrow$ (i) Take an arbitrary normal state $\varphi$. Then, from the definition of $\mathbb{E}$ we have

$$
\varphi \circ \mathbb{E}=\left(R \circ m_{T}\right)(\varphi)=m_{T}(\varphi)_{n} \text { - the normal part of } m_{T}(\varphi) .
$$

Hence

$$
m_{T}(\varphi)_{n}(\mathbb{1})=\varphi(\mathbb{E}(\mathbb{1}))=\varphi(\mathbb{1})=1,
$$

so for the singular part we obtain

$$
m_{T}(\varphi)_{s}(\mathbb{1})=0
$$

which means that $m_{T}(\varphi)_{s}=0$, i.e. $m_{T}(\varphi)$ is normal. Since the normal states linearly span $\mathcal{M}_{*}$, it follows that $\left(T_{t}\right)$ is mean ergodic.

In [24, Corollary 13], it was shown that there is at most one positive normal norm-one projection $\mathbb{E}$ such that

(i) $\left\{\varphi \circ \mathbb{E}: \varphi \in \mathcal{M}_{*}\right\}=\operatorname{Fix} T$,

(ii) $\mathbb{E}(\mathcal{M})=\operatorname{Fix} T^{*}$.

Consequently, if the quasi-ergodic projection $\mathbb{E}$ associated with invariant mean $m$ satisfies the conditions of Proposition 3 , then $\mathbb{E}$ is unique; in other words it is independent of $m$. In this case $\mathbb{E}$ will be called the ergodic projection for $\left(T_{t}\right)$, and will be denoted by $\mathbb{E}^{T}$. From formula (10), we obtain, by virtue of the relation $\mathbb{E}^{T}=\left(m_{T} \mid \mathcal{M}_{*}\right)^{*}$,

$$
\varphi\left(\mathbb{E}^{T} x\right)=\lim _{\gamma} \varphi\left(\sum_{i=1}^{n_{\gamma}} \lambda_{i}^{\gamma} T_{t_{i}^{\gamma}}^{*} x\right), \quad x \in \mathcal{M}, \varphi \in \mathcal{M}_{*},
$$

which means that $\mathbb{E}^{T}$ is a point- $\sigma$-weak limit of convex combinations of some $T_{t}^{*}$, i.e.

$$
\mathbb{E}^{T} \in \overline{\operatorname{conv}\left\{T_{t}^{*}: t \in G\right\}},
$$

where the closure is in the point- $\sigma$-weak topology.

Remark 4. In general, given a quantum dynamical semigroup $\left(S_{t}: t \in\right.$ $G)$, by an ergodic projection for this semigroup is meant a bounded linear map $\mathbb{E}: \mathcal{M} \rightarrow \mathcal{M}$ such that

(i) $S_{t} \mathbb{E}=\mathbb{E} S_{t}=\mathbb{E}$ for each $t \in G$,

(ii) $\mathbb{E} \in \overline{\operatorname{conv}\left\{S_{t}: t \in G\right\}}$, where the closure is in the point- $\sigma$-weak topology.

It follows that $\mathbb{E}$ is a projection onto the fixed-point space of $\left(S_{t}\right)$. However, it need not be normal. Ergodic projections in this general setting were investigated in [25] and [27]. 
In [30, Definition III.7.1], a general definition of mean ergodicity is given which, in our setting, reads as follows. The semigroup $\left(T_{t}: t \in G\right)$ is said to be mean ergodic if there is an element $P \in \overline{\operatorname{conv}\left\{T_{t}: t \in G\right\}}$, the closure being in the point-norm (or, equivalently, point-weak topology), such that we have

$$
P T_{t}=T_{t} P=P \text { for each } t \in G .
$$

Proposition 4. The two definitions of mean ergodicity (that of [30, Definition III.7.1] and ours) are equivalent.

Proof: Suppose first that $\left(T_{t}\right)$ is mean ergodic in our sense, and let $m$ be an arbitrary invariant mean on $\ell^{\infty}(G)$. Then $m_{T} \mid \mathcal{M}_{*}: \mathcal{M}_{*} \rightarrow \mathcal{M}_{*}$,

$$
T_{t} m_{T}=m_{T} T_{t}=m_{T} \quad \text { for each } t \in G,
$$

and on account of equation (10), $m_{T} \in \overline{\operatorname{conv}\left\{T_{t}: t \in G\right\}}$ which shows that $\left(T_{t}\right)$ is mean ergodic in the sense of [30, Definition III.7.1].

Now let $\left(T_{t}\right)$ be mean ergodic in the sense of [30, Definition III.7.1], and let $P \in \overline{\operatorname{conv}\left\{T_{t}: t \in G\right\}}$ satisfy equation (14). For the adjoint operator $P^{*}$ we have: $P^{*}: \mathcal{M} \rightarrow \mathcal{M}, P^{*}$ is normal,

$$
T_{t}^{*} P^{*}=P^{*} T_{t}^{*}=P^{*} \quad \text { for each } t \in G,
$$

and $P^{*} \in \overline{\operatorname{conv}\left\{T_{t}^{*}: t \in G\right\}}$, where the closure is in the point- $\sigma$-weak topology. Thus, in line with the terminology of Remark $4, P^{*}$ is an ergodic projection for the quantum dynamical semigroup $\left(T_{t}^{*}: t \in G\right)$.

Let $m$ be an arbitrary invariant mean on $\ell^{\infty}(G)$. In [17, p. 828], a bounded projection $\widetilde{\mathbb{E}}$ of norm one from $\mathcal{M}$ onto Fix $T^{*}$ was constructed such that

$$
T_{t}^{*} \widetilde{\mathbb{E}}=\widetilde{\mathbb{E}} T_{t}^{*}=\widetilde{\mathbb{E}} \text { for each } t \in G,
$$

and $\widetilde{\mathbb{E}} \in \overline{\operatorname{conv}\left\{T_{t}^{*}: t \in G\right\}}$, the closure being in the point- $\sigma$-weak topology, so $\widetilde{\mathbb{E}}$ is also an ergodic projection for the quantum dynamical semigroup $\left(T_{t}^{*}: t \in G\right)$. (Actually, the ergodic projection $\widetilde{\mathbb{E}}$ was constructed in $[\mathbf{1 7}]$ in a more general case of a semigroup of contractions on $\mathcal{M}$.) Let

$$
\widetilde{\mathbb{E}}=\widetilde{\mathbb{E}}_{n}+\widetilde{\mathbb{E}}_{s}
$$

be the decomposition of $\widetilde{\mathbb{E}}$ into the normal and singular parts. By virtue of [27, Theorem 2], we have

$$
\widetilde{\mathbb{E}}_{n}=P^{*} .
$$

Hence

$$
\mathbb{1}=\widetilde{\mathbb{E}}(\mathbb{1})=\widetilde{\mathbb{E}}_{n}(\mathbb{1})+\widetilde{\mathbb{E}}_{s}(\mathbb{1})=P^{*}(\mathbb{1})+\widetilde{\mathbb{E}}_{s}(\mathbb{1})=\mathbb{1}+\widetilde{\mathbb{E}}_{s}(\mathbb{1}),
$$


which yields $\widetilde{\mathbb{E}}_{s}(\mathbb{1})=0$, so $\widetilde{\mathbb{E}}_{n}(\mathbb{1})=\mathbb{1}$. Let $\mathbb{E}$ be the quasi-ergodic projection associated with the mean $m$. In $[\mathbf{1 7}$, p. 828], it was shown that

$$
\mathbb{E}=\widetilde{\mathbb{E}}_{n}
$$

Thus $\mathbb{E}(\mathbb{1})=\widetilde{\mathbb{E}}_{n}(\mathbb{1})=\mathbb{1}$, and on account of Proposition $3,\left(T_{t}\right)$ is mean ergodic in our sense.

The following lemma shows a connection between mean ergodicity and generalised averages.

Lemma 5. Let $\left(T_{t}\right)$ be a mean ergodic Markov semigroup, and let $\left\{A_{\gamma}\right.$ : $\gamma \in \Gamma\}$ be a generalised average on $G$. Then, for each $\varphi \in \mathcal{M}_{*}$, the net $\left\{\left(A_{\gamma}\right)_{T}(\varphi): \gamma \in \Gamma\right\}$ converges weakly to $m_{T}(\varphi)=\varphi \circ \mathbb{E}^{T} \in \mathrm{Fix} T$.

Proof: It is enough to prove the statement for $\|\varphi\| \leqslant 1$. The net $\left\{\left(A_{\gamma}\right)_{T}(\varphi)\right.$ : $\gamma \in \Gamma\} \subset \mathcal{M}^{*}$ is norm-bounded, and thus relatively weak ${ }^{*}$-compact. Let $\widetilde{\varphi} \in \mathcal{M}^{*}$ be a limit point of this net. Then there is a subnet $\left\{\left(A_{\gamma^{\prime}}\right)_{T}(\varphi)\right\}$ convergent to $\widetilde{\varphi}$ weakly* ${ }^{*}$. Let $m^{\prime}$ be an invariant mean associated with the net $\left\{A_{\gamma^{\prime}}\right\}$. Then for the ergodic projection $\mathbb{E}^{T}$ we have $\mathbb{E}^{T}=\left(m_{T}^{\prime} \mid \mathcal{M}_{*}\right)^{*}$, and thus

$$
m_{T}^{\prime}(\varphi)=\varphi \circ \mathbb{E}^{T}
$$

Consequently, we obtain for each $x \in \mathcal{M}$,

$$
\begin{aligned}
\widetilde{\varphi}(x) & =\lim _{\gamma^{\prime}}\left(A_{\gamma^{\prime}}\right)_{T}(\varphi)(x)=\lim _{\gamma^{\prime}} A_{\gamma^{\prime}}\left(\left[t \mapsto\left(T_{t} \varphi\right)(x)\right]\right) \\
& =m^{\prime}\left(\left[t \mapsto\left(T_{t} \varphi\right)(x)\right]\right)=m_{T}^{\prime}(\varphi)(x)=\left(\varphi \circ \mathbb{E}^{T}\right)(x),
\end{aligned}
$$

which means that all the weak ${ }^{*}$-limit points of $\left\{\left(A_{\gamma}\right)_{T}(\varphi): \gamma \in \Gamma\right\}$ coincide with $\varphi \circ \mathbb{E}^{T}$. It follows that the net $\left\{\left(A_{\gamma}\right)_{T}(\varphi): \gamma \in \Gamma\right\}$ itself converges weakly to $\varphi \circ \mathbb{E}^{T} \in \operatorname{Fix} T$.

In general, we have the following result which shows the uniqueness of a limit of generalised averages.

Lemma 6. Let $\left\{A_{\gamma}: \gamma \in \Gamma\right\}$ and $\left\{A_{\theta}^{\prime}: \theta \in \Theta\right\}$ be generalised averages on $G$, and let $\varphi \in \mathcal{M}_{*}$ be such that $\lim _{\gamma \in \Gamma}\left(A_{\gamma}\right)_{T}(\varphi)$ and $\lim _{\theta \in \Theta}\left(A_{\theta}^{\prime}\right)_{T}(\varphi)$ exist. Then

$$
\lim _{\gamma \in \Gamma}\left(A_{\gamma}\right)_{T}(\varphi)=\lim _{\theta \in \Theta}\left(A_{\theta}^{\prime}\right)_{T}(\varphi) .
$$

Proof: Let $m$ be an invariant mean associated with $\left\{A_{\gamma}: \gamma \in \Gamma\right\}$, and let $m^{\prime}$ be an invariant mean associated with $\left\{A_{\theta}^{\prime}: \theta \in \Theta\right\}$. Then on account of Lemma 2 we have

$$
\lim _{\gamma \in \Gamma}\left(A_{\gamma}\right)_{T}(\varphi)=m_{T}(\varphi)=m_{T}^{\prime}(\varphi)=\lim _{\theta \in \Theta}\left(A_{\theta}^{\prime}\right)_{T}(\varphi) .
$$


It is interesting to observe that in some cases the converse to Lemma 5 is also true. Namely, suppose that $\left\{A_{\gamma}: \gamma \in \Gamma\right\}$ is a generalised average such that there is a subnet in the form of a sequence $\left\{A_{\gamma_{1}}, A_{\gamma_{2}}, \ldots\right\}$, and that for each $\varphi \in \mathcal{M}_{*}$ we have $\left(A_{\gamma}\right)_{T}(\varphi) \in \mathcal{M}_{*}$ (note that the nets of Cesàro means, Borel means, and Abel means have these two properties). If for each $\varphi \in \mathcal{M}_{*}$ the net $\left\{\left(A_{\gamma}\right)_{T}(\varphi): \gamma \in \Gamma\right\}$ converges weakly, then $\left(T_{t}\right)$ is mean ergodic. Indeed, let $m$ be an invariant mean associated with $\left\{A_{\gamma}: \gamma \in \Gamma\right\}$. Then, for each $\varphi \in \mathcal{M}_{*}$, we have

$$
m_{T}(\varphi)=\lim _{\gamma}\left(A_{\gamma}\right)_{T}(\varphi)=\lim _{n \rightarrow \infty}\left(A_{\gamma_{n}}\right)_{T}(\varphi),
$$

and since $\left(A_{\gamma_{n}}\right)_{T}(\varphi) \in \mathcal{M}_{*}$, we infer on account of [32, Theorem III.5.1] that $m_{T}(\varphi)$ as a weak limit of a sequence of elements in $\mathcal{M}_{*}$ is also in $\mathcal{M}_{*}$. Thus $\left(T_{t}\right)$ is mean ergodic.

To obtain an equivalent condition for the ergodicity of $\left(T_{t}\right)$ in terms of generalised averages, we need one more notion. Put

$$
p_{r}=\sup \left\{s(\varphi): \varphi \in(\operatorname{Fix} T)^{+}\right\} .
$$

$p_{r}$ is called the recurrent projection. Let $\mathbb{E}$ be a quasi-ergodic projection (associated with some $m$ ). For each $\varphi \in(\operatorname{Fix} T)^{+}$, we have on account of property (i) of a quasi-ergodic projection

$$
\varphi \circ \mathbb{E}=\varphi .
$$

Thus [33, Lemma 1] yields the inequality

$$
\mathbb{E} \mathrm{s}(\varphi) \geqslant \mathrm{s}(\varphi) \text { for each } \varphi \in(\operatorname{Fix} T)^{+},
$$

so

$$
\mathbb{E} p_{r} \geqslant p_{r} .
$$

We have the following generalisation of Proposition IV.3.3 in [14] which, at the same time, shows the usefulness of the notions of recurrent projection and the limit along a semigroup.

Theorem 7. Let $\left(T_{t}\right)$ be a Markov semigroup. The following conditions are equivalent:

(i) $\left(T_{t}\right)$ is mean ergodic,

(ii) for every generalised average $\left\{A_{\gamma}: \gamma \in \Gamma\right\}$ on $G$, we have

$$
\lim _{\gamma}\left(A_{\gamma}\right)_{T}(\varphi)\left(p_{r}\right)=\varphi(\mathbb{1}), \quad \varphi \in \mathcal{M}_{*},
$$

(iii) for some generalised average $\left\{A_{\gamma}: \gamma \in \Gamma\right\}$ on $G$, we have

$$
\lim _{\gamma}\left(A_{\gamma}\right)_{T}(\varphi)\left(p_{r}\right)=\varphi(\mathbb{1}), \quad \varphi \in \mathcal{M}_{*},
$$

$$
\text { strong operator- } \lim _{t \in G} T_{t}^{*} p_{r}=\mathbb{1} \text {. }
$$


Proof: (i) $\Longrightarrow$ (ii) Let $\left\{A_{\gamma}: \gamma \in \Gamma\right\}$ be a generalised average on $G$, and let $m$ be an invariant mean associated with it. Since $\left(T_{t}\right)$ is mean ergodic we have, for the ergodic projection $\mathbb{E}^{T}$ and each $\varphi \in \mathcal{M}_{*}^{+}$,

$$
m_{T}(\varphi)(\mathbb{1})=\varphi\left(\mathbb{E}^{T}(\mathbb{1})\right)=\varphi(\mathbb{1}) .
$$

From Lemma 5, it follows that there exists

$$
\lim _{\gamma}\left(A_{\gamma}\right)_{T}(\varphi)=m_{T}(\varphi)
$$

Since $m_{T}(\varphi) \in \operatorname{Fix} T$, we have $\mathrm{s}\left(m_{T}(\varphi)\right) \leqslant p_{r}$. Hence

$$
\lim _{\gamma}\left(A_{\gamma}\right)_{T}(\varphi)\left(p_{r}\right)=m_{T}(\varphi)\left(p_{r}\right)=m_{T}(\varphi)(\mathbb{1})=\varphi(\mathbb{1}) .
$$

Since every element in $\mathcal{M}_{*}$ is a linear combination of four positive functionals, we obtain (ii).

(ii) $\Longrightarrow$ (iii) Obvious.

(iii) $\Longrightarrow$ (i) Let $\left\{A_{\gamma}: \gamma \in \Gamma\right\}$ be a generalised average on $G$ satisfying (15), and let $m$ be an invariant mean associated with it. Take an arbitrary $\varphi \in$ $\mathcal{M}_{*}^{+}$and let $f^{p_{r}} \in \ell^{\infty}(G)$ be as before, i.e.

$$
f^{p_{r}}(t)=\left(T_{t} \varphi\right)\left(p_{r}\right), \quad t \in G .
$$

Then

$$
A_{\gamma}\left(f^{p_{r}}\right)=\left(A_{\gamma}\right)_{T}(\varphi)\left(p_{r}\right) \underset{\gamma}{\longrightarrow} \varphi(\mathbb{1}) .
$$

Thus according to the definition of the associated mean we obtain

$$
m\left(f^{p_{r}}\right)=\varphi(\mathbb{1}) .
$$

On the other hand, we have

$$
m\left(f^{p_{r}}\right)=m_{T}(\varphi)\left(p_{r}\right),
$$

so

$$
m_{T}(\varphi)\left(p_{r}\right)=\varphi(\mathbb{1}) .
$$

Let $\mathbb{E}$ be the quasi-ergodic projection associated with $m$. On account of formula (12), for the decomposition of $m_{T}(\varphi)$ into the normal and singular parts we have

$$
m_{T}(\varphi)=\varphi \circ \mathbb{E}+m_{T}(\varphi)_{s} .
$$

For $x \in \operatorname{Fix} T^{*}$, we have

$$
m_{T}(\varphi)(x)=\varphi(x)
$$


and thus for arbitrary $x \in \mathcal{M}$ we obtain, taking into account that $\mathbb{E} x \in$ Fix $T^{*}$,

$$
\begin{aligned}
\varphi(\mathbb{E} x) & =m_{T}(\varphi)(x)=(\varphi \circ \mathbb{E})(\mathbb{E} x)+m_{T}(\varphi)_{s}(\mathbb{E} x) \\
& =\varphi(\mathbb{E} x)+m_{T}(\varphi)_{s}(\mathbb{E} x),
\end{aligned}
$$

which yields

$$
m_{T}(\varphi)_{s}(\mathbb{E} x)=0
$$

i.e.

$$
m_{T}(\varphi)_{s} \circ \mathbb{E}=0 .
$$

Since $\mathbb{E} p_{r} \geqslant p_{r}$, we get

$$
0=m_{T}(\varphi)_{s}\left(\mathbb{E} p_{r}\right) \geqslant m_{T}(\varphi)_{s}\left(p_{r}\right),
$$

SO

$$
m_{T}(\varphi)_{s}\left(p_{r}\right)=0 .
$$

Equalities (16), (17), and (18) yield

$$
\varphi(\mathbb{1})=m_{T}(\varphi)\left(p_{r}\right)=(\varphi \circ \mathbb{E})\left(p_{r}\right)+m_{T}(\varphi)_{s}\left(p_{r}\right)=\varphi\left(\mathbb{E} p_{r}\right),
$$

and since $\varphi \in \mathcal{M}_{*}^{+}$was arbitrary, this implies

$$
\mathbb{E} p_{r}=\mathbb{1} \text {. }
$$

$\mathbb{E}$ is a projection, hence we obtain

$$
\mathbb{E}(\mathbb{1})=\mathbb{E}\left(\mathbb{E} p_{r}\right)=\mathbb{E} p_{r}=\mathbb{1},
$$

which by virtue of Proposition 3 , shows that $\left(T_{t}\right)$ is mean ergodic.

Before proving the equivalence of condition (iv) with the remaining ones, we make the following simple but crucial observation (in fact, of the same nature as that before this theorem). For each $\varphi \in(\text { Fix } T)^{+}$and each $t \in G$, we have

$$
\varphi \circ T_{t}^{*}=\varphi .
$$

Thus [33, Lemma 1] yields the inequality

$$
T_{t}^{*} \mathrm{~s}(\varphi) \geqslant \mathrm{s}(\varphi) \text { for each } \varphi \in(\operatorname{Fix} T)^{+},
$$

so

$$
T_{t}^{*} p_{r} \geqslant p_{r}
$$

Consequently, for arbitrary $s, t \in G$, we have

$$
T_{s+t}^{*} p_{r}=T_{t}^{*}\left(T_{s}^{*} p_{r}\right) \geqslant T_{t}^{*} p_{r},
$$

which means that the net $\left\{T_{t}^{*} p_{r}: t \in G\right\}$ is increasing. Thus there exists a strong operator limit (= the supremum of the net)

$$
\lim _{t \in G} T_{t}^{*} p_{r}=z .
$$


In particular, for arbitrary $\varphi \in \mathcal{M}_{*}$, we have

$$
\lim _{t \in G}\left(T_{t} \varphi\right)\left(p_{r}\right)=\lim _{t \in G} \varphi\left(T_{t}^{*} p_{r}\right)=\varphi(z) .
$$

Let $m$ be an arbitrary invariant mean. For the function $f^{p_{r}}$, we have

$$
\lim _{t \in G} f^{p_{r}}(t)=\lim _{t \in G}\left(T_{t} \varphi\right)\left(p_{r}\right)=\varphi(z),
$$

and thus, on account of relation (4), we obtain the equality

$$
m_{T}(\varphi)\left(p_{r}\right)=m\left(f^{p_{r}}\right)=\lim _{t \in G} f^{p_{r}}(t)=\varphi(z) .
$$

(ii) $\Longrightarrow$ (iv) Let $\Gamma$ be an arbitrary directed set and let $m$ be an arbitrary invariant mean. For each $\gamma \in \Gamma$, put

$$
A_{\gamma}(f)=m(f), \quad f \in \ell^{\infty}(G) .
$$

Then $\left\{A_{\gamma}: \gamma \in \Gamma\right\}$ is a (constant) generalised average, and for every $\varphi \in$ $\mathcal{M}_{*}$, we have

$$
\varphi(\mathbb{1})=\lim _{\gamma \in \Gamma}\left(A_{\gamma}\right)_{T}(\varphi)\left(p_{r}\right)=m_{T}(\varphi)\left(p_{r}\right)=\varphi(z),
$$

which yields

$$
\mathbb{1}=z=\lim _{t \in G} T_{t}^{*} p_{r}
$$

(iv) $\Longrightarrow$ (iii) We take the same particular generalised average as in the previous implication and obtain, on account of equality (19) and the equality $z=\mathbb{1}$,

$$
\lim _{\gamma \in \Gamma}\left(A_{\gamma}\right)_{T}(\varphi)\left(p_{r}\right)=m_{T}(\varphi)\left(p_{r}\right)=\lim _{t \in G} f^{p_{r}}(t)=\varphi(z)=\varphi(\mathbb{1})
$$

for each $\varphi \in \mathcal{M}_{*}$, so the conclusion follows.

As an immediate corollary we obtain

Corollary 8. Let $(\operatorname{Fix} T)^{+}$be a faithful family. Then $\left(T_{t}\right)$ is mean ergodic.

Indeed, for $(\operatorname{Fix} T)^{+}$faithful, we have $p_{r}=\mathbb{1}$ and thus $T_{t}^{*} p_{r}=\mathbb{1}$.

A subsemigroup $H$ of $G$ is said to be cofinal with $G$ if for every $t \in G$ there is $s \in H$ such that $t \leqslant s$.

Theorem 9. Let $H$ be a cofinal subsemigroup of $G$. If $\left(T_{t}: t \in G\right)$ is mean ergodic, then $\left(T_{t}: t \in H\right)$ is also mean ergodic.

Proof: Let $p_{r}$ be the recurrent projection for $\left(T_{t}: t \in G\right)$, and let $q_{r}$ be the recurrent projection for $\left(T_{t}: t \in H\right)$. Since every $\left(T_{t}: t \in G\right)$-invariant state is also $\left(T_{t}: t \in H\right)$-invariant, we have

$$
p_{r} \leqslant q_{r} .
$$


From the assumption that $H$ is cofinal with $G$, it follows that the net $\left\{T_{t}^{*}: t \in H\right\}$ is a subnet of $\left\{T_{t}^{*}: t \in G\right\}$, so from Theorem 7(iv) we get

$$
\mathbb{1}=\lim _{t \in G} T_{t}^{*} p_{r}=\lim _{t \in H} T_{t}^{*} p_{r} \leqslant \lim _{t \in H} T_{t}^{*} q_{r} \leqslant \mathbb{1} .
$$

Thus

$$
\lim _{t \in H} T_{t}^{*} q_{r}=\mathbb{1},
$$

and Theorem 7(iv) gives the claim.

As a corollary we obtain the following generalisation of Theorem 12 in $[\mathbf{9}]$.

Corollary 10. Let $\left(T_{t}: t \in G\right)$ be mean ergodic, where $G=\mathbb{Z}_{+}$or $G=[0, \infty)$. Then, for each $t \in G$, the semigroup $\left(T_{t}^{n}: n=0,1, \ldots\right)$ is mean ergodic.

Assume that $\left(T_{t}\right)$ is mean ergodic, and let $\mathbb{E}^{T}$ be the ergodic projection. We define a product ' $\bullet$ ' on $\left(\text { Fix } T^{*}\right)^{h}$ by

$$
x \bullet y=\mathbb{E}^{T}(x \circ y),
$$

where $\circ$ stands for the Jordan product on $\mathbb{B}(\mathcal{H})$, i.e. $x \circ y=1 / 2(x y+y x)$. Then, since $\left(\text { Fix } T^{*}\right)^{h}=\mathbb{E}^{T}\left(\mathcal{M}^{h}\right)$, it follows from [4, Corollary 1.5] that $\left(\left(\operatorname{Fix} T^{*}\right)^{h}, \bullet\right)$ is a JW-algebra, by which is meant that it has an isometric Jordan representation as a weakly closed Jordan algebra of bounded selfadjoint operators on some Hilbert space. In particular, $\left(\left(\operatorname{Fix} T^{*}\right)^{h}, \bullet\right)$ has plenty of projections and for each such projection $e$ we have

$$
e=e \bullet e=\mathbb{E}^{T} e^{2} \text {. }
$$

Moreover, from the formula

$$
\mathbb{E}^{T}\left(x \circ \mathbb{E}^{T} y\right)=\mathbb{E}^{T}(x \circ y), \quad x \in\left(\operatorname{Fix} T^{*}\right)^{h}, y \in \mathcal{M}^{h},
$$

proven in $[\mathbf{4}$, Lemma 1.1], we infer that

$$
e=e \bullet(e \bullet e)=\mathbb{E}^{T}(e \circ(e \bullet e))=\mathbb{E}^{T}\left(e \circ \mathbb{E}^{T} e^{2}\right)=\mathbb{E}^{T}\left(e \circ e^{2}\right)=\mathbb{E}^{T} e^{3},
$$

and by induction

$$
e=\mathbb{E}^{T} e^{n}
$$

for each positive integer $n$. (Note that our notation concerning powers refers to the customary product of operators in $\mathbb{B}(\mathcal{H})$.)

Let $\pi$ be an isometric Jordan representation of $\left(\operatorname{Fix} T^{*}\right)^{h}$ on some Hilbert space. Since

$$
\operatorname{Fix} T^{*}=\left(\operatorname{Fix} T^{*}\right)^{h} \oplus i\left(\operatorname{Fix} T^{*}\right)^{h},
$$


we can define an isometric Jordan representation $\widehat{\pi}$ of Fix $T^{*}$ by the formula

$$
\widehat{\pi}(x+i y)=\pi(x)+i \pi(y), \quad x, y \in\left(\operatorname{Fix} T^{*}\right)^{h} .
$$

Then $\widehat{\pi}\left(\operatorname{Fix} T^{*}\right)$ is a weakly closed *-algebra of bounded operators on a Hilbert space, closed with respect to the Jordan product, i.e. $\widehat{\pi}\left(\mathrm{Fix} T^{*}\right)$ is a $\mathrm{JW}^{*}$-algebra.

Now we want to obtain a similar result for strongly positive maps. Our first observation is the following:

Lemma 11. A convex combination of strongly positive unital maps between unital $C^{*}$-algebras is strongly positive.

Proof: First, we shall show that for any $x, y \in \mathbb{B}(\mathcal{H})$ and any $\lambda \in[0,1]$, we have

$$
|\lambda x+(1-\lambda) y|^{2} \leqslant \lambda|x|^{2}+(1-\lambda)|y|^{2} .
$$

(This property could be called the operator convexity of the function $x \mapsto$ $|x|^{2}$. However, this name is employed usually for functions defined on subsets of the real line and applied to selfadjoint operators.)

From the inequality

$$
(x-y)^{*}(x-y) \geqslant 0,
$$

it follows that

$$
|x|^{2}+|y|^{2} \geqslant x^{*} y+y^{*} x
$$

We have

$$
|\lambda x+(1-\lambda) y|^{2}=\lambda^{2}|x|^{2}+(1-\lambda)^{2}|y|^{2}+\lambda(1-\lambda)\left(x^{*} y+y^{*} x\right),
$$

hence

$\lambda|x|^{2}+(1-\lambda)|y|^{2}-|\lambda x+(1-\lambda) y|^{2}=\lambda(1-\lambda)\left(|x|^{2}+|y|^{2}-\left(x^{*} y+y^{*} x\right)\right) \geqslant 0$, showing the claim.

Now let $\Phi$ and $\Psi$ be strongly positive unital maps. For arbitrary $0 \leqslant \lambda \leqslant 1$, we obtain

$$
\begin{aligned}
(\lambda \Phi+ & (1-\lambda) \Psi)\left(x^{*}\right)(\lambda \Phi+(1-\lambda) \Psi)(x) \\
& =\mid\left(\lambda \Phi(x)+\left.(1-\lambda) \Psi(x)\right|^{2} \leqslant \lambda|\Phi(x)|^{2}+(1-\lambda)|\Psi(x)|^{2}\right. \\
& \leqslant \lambda \Phi\left(x^{*} x\right)+(1-\lambda) \Psi\left(x^{*} x\right)=(\lambda \Phi+(1-\lambda) \Psi)\left(x^{*} x\right),
\end{aligned}
$$

finishing the proof.

Let now $\left(T_{t}\right)$ be a mean ergodic Markov semigroup on $\mathcal{M}_{*}$ with the adjoint semigroup being strongly positive. On account of relation (13), the ergodic projection $\mathbb{E}^{T}$ is strongly positive. 
We need a result which is a slight generalisation of one in [1]. Namely, it was proven there that if $\mathcal{M}$ is a von Neumann algebra and $\mathbb{E}$ is a completely positive norm-one projection defined on $\mathcal{M}$, then $\mathbb{E}(\mathcal{M})$ is a $\mathrm{W}^{*}$-algebra under the product '.' defined by

$$
x \cdot y=\mathbb{E}(x y), \quad x, y \in \mathbb{E}(\mathcal{M})
$$

(cf. [1, Theorem 3.1 and Corollary 3.2]). However, it can be shown that the same is true if complete positivity is weakened to strong positivity (cf. [28]). Consequently, if the semigroup $\left(T_{t}: t \in G\right)$ is mean ergodic with strongly positive adjoint semigroup, then $\mathrm{Fix} T^{*}$ is a $\mathrm{W}^{*}$-algebra under the above product. In other words, a $\mathrm{C}^{*}$-algebra which is a dual Banach space [32, Section III.3].

The isometrical isomorphism between $(\operatorname{Fix} T)^{*}$ and $\mathbb{E}(\mathcal{M})$ was proven in $[\mathbf{1 7}$, Theorem 3] in a more general setup assuming only the contractivity of $\left(T_{t}\right)$. For the sake of completeness we give a proof below.

Lemma 12. Let $\mathbb{E}$ be a quasi-ergodic projection for a Markov semigroup $\left(T_{t}\right)$ on $\mathcal{M}_{*}$. Then

$$
\mathbb{E}(\mathcal{M}) \simeq(\operatorname{Fix} T)^{*} \text { and } \mathbb{E}\left(\mathcal{M}^{h}\right) \simeq\left((\operatorname{Fix} T)^{h}\right)^{*} .
$$

Proof: It is enough to prove only the first relation in (23), the proof of the second being analogous.

Each $x \in \mathbb{E}(\mathcal{M})$ can be considered as a bounded linear functional on $\mathcal{M}_{*}$. Define a linear map $\Phi: \mathbb{E}(\mathcal{M}) \rightarrow(\operatorname{Fix} T)^{*}$ by the formula

$$
\Phi x=x \mid \operatorname{Fix} T .
$$

Clearly,

$$
\|\Phi x\|=\|x \mid \operatorname{Fix} T\| \leqslant\|x\| .
$$

On the other hand, since $x=\mathbb{E} x, \varphi \circ \mathbb{E} \in \mathcal{M}_{*}$ for any $\varphi \in \mathcal{M}_{*}$, and $\|\varphi \circ \mathbb{E}\| \leqslant\|\varphi\|$, we obtain

$$
\begin{aligned}
\|x\| & =\|\mathbb{E} x\|=\sup \left\{|\varphi(\mathbb{E} x)|:\|\varphi\| \leqslant 1, \varphi \in \mathcal{M}_{*}\right\} \\
& =\sup \left\{|(\varphi \circ \mathbb{E})(x)|:\|\varphi\| \leqslant 1, \varphi \in \mathcal{M}_{*}\right\} \\
& \leqslant \sup \{|\psi(x)|:\|\psi\| \leqslant 1, \psi \in \operatorname{Fix} T\}=\|x \mid \operatorname{Fix} T\|=\|\Phi x\|,
\end{aligned}
$$

showing that $\Phi$ is an isometry. For any $h \in(\operatorname{Fix} T)^{*}$, let $\widehat{h}$ be a HahnBanach extension of $h$ to $\left(\mathcal{M}_{*}\right)^{*}=\mathcal{M}$. Hence $\widehat{h}=x$ for some $x \in \mathcal{M}$, and clearly $x \mid \operatorname{Fix} T=h$, i.e. $\langle h, \varphi\rangle=\varphi(x)$ for each $\varphi \in \operatorname{Fix} T$. For $\mathbb{E} x \in \mathbb{E}(\mathcal{M})$, we have

$$
(\mathbb{E} x \mid \operatorname{Fix} T)(\varphi)=\varphi(\mathbb{E} x)=\varphi(x)=\langle h, \varphi\rangle, \quad \varphi \in \operatorname{Fix} T,
$$

so

$$
h=\mathbb{E} x \mid \operatorname{Fix} T=\Phi(\mathbb{E} x),
$$

which proves that $\Phi$ is surjective. 
Let $\mathbb{E}^{T}$ be the ergodic projection for a mean ergodic Markov semigroup. Then $\mathbb{E}^{T}(\mathcal{M})=$ Fix $T^{*}$, and $\mathbb{E}^{T}\left(\mathcal{M}^{h}\right)=\left(\operatorname{Fix} T^{*}\right)^{h}$, so we get on account of the above lemma

$$
\begin{aligned}
\operatorname{Fix} T^{*} & =\mathbb{E}^{T}(\mathcal{M}) \simeq(\operatorname{Fix} T)^{*}, \\
\left(\operatorname{Fix} T^{*}\right)^{h} & =\mathbb{E}^{T}\left(\mathcal{M}^{h}\right) \simeq\left((\operatorname{Fix} T)^{h}\right)^{*} .
\end{aligned}
$$

In particular, $(\operatorname{Fix} T)^{h}$ is the predual of the JW-algebra $\left(\left(\operatorname{Fix} T^{*}\right)^{h}, \bullet\right)$, and if the adjoint semigroup is strongly positive, then Fix $T$ is the predual of the $\mathrm{W}^{*}$-algebra Fix $T^{*}$.

\section{Ergodic theorems and asymptotic stability}

We start with some basic facts from the theory of Jordan algebras (see [15] for a detailed account of this theory).

Proposition 13. Let $\mathfrak{A}$ be a $J W$-algebra such that every resolution of identity in $\mathfrak{A}$ is finite. Then $\mathfrak{A}$ is a finite direct sum of $J W$-factors of type $\mathrm{I}_{n_{i}}$ with all the $n_{i}$ finite.

Proof: Since every resolution of identity in $\mathfrak{A}$ is finite, we may assume that

$$
\sum_{i=1}^{n} e_{i}=\mathbb{1}
$$

is such a resolution into minimal projections; moreover, we may also assume that $\mathfrak{A}$ is represented as a weak-operator closed algebra of bounded selfadjoint operators on a Hilbert space, closed with respect to the Jordan product 'o'. For every element $a$ of $\mathfrak{A}$, we define on $\mathfrak{A}$ the operator $T_{a}$ by the formula

$$
T_{a} b=a \circ b, \quad b \in \mathfrak{A} .
$$

Two elements $a, b \in \mathfrak{A}$ are said to operator commute if $T_{a} T_{b}=T_{b} T_{a}$. It is easily seen that $a b=b a$ implies the operator commuting of $a$ and $b$. The centre $\mathcal{Z}(\mathfrak{A})$ of $\mathfrak{A}$ is defined as the set of these elements in $\mathfrak{A}$ which operator commute with each element in $\mathfrak{A}$. An operator $s \in \mathfrak{A}$ is called a symmetry if $s^{2}=\mathbb{1}$. On account of [15, Lemma 4.3.2], we have

$$
\mathcal{Z}(\mathfrak{A})=\{a \in \mathfrak{A}: a s=s a \text { for every symmetry } s \in \mathfrak{A}\} .
$$

Since each projection $p$ in $\mathfrak{A}$ has the form

$$
p=\frac{s+\mathbb{1}}{2},
$$

where $s$ is a symmetry, we infer that $\mathcal{Z}(\mathfrak{A})$ is the set of operators in $\mathfrak{A}$ which commute with all projections in $\mathfrak{A}$, and since $\mathfrak{A}$ is a JW-algebra, $\mathcal{Z}(\mathfrak{A})$ is the set of operators in $\mathfrak{A}$ which commute with all operators in $\mathfrak{A}$. 
Since every resolution of identity in $\mathfrak{A}$ is finite, we obtain that its centre has a finite family of pairwise orthogonal minimal projections $z_{k}$, for $k=1, \ldots, m$, such that $\sum_{k=1}^{m} z_{k}=\mathbb{1}$. For the minimal projections $e_{i}$ and each $z_{k}, e_{i} z_{k}$ is a projection too, so the minimality of $e_{i}$ yields $e_{i} z_{k}=e_{i}$ or 0 . Consequently, each $z_{k}$ is a finite sum of some $e_{i}$. For a central projection $z$ of $\mathfrak{A}$, put

$$
\mathfrak{A}_{z}=\{z a \mid z(\mathcal{H}): a \in \mathfrak{A}\} .
$$

We have

$$
\mathfrak{A}=\mathfrak{A}_{z_{1}} \oplus \cdots \oplus \mathfrak{A}_{z_{m}},
$$

and since the projections $z_{k}$ are minimal central, each $\mathfrak{A}_{z_{k}}$ is a factor of type $\mathrm{I}_{n}$. Since $z_{k}$ is a finite sum of minimal projections, it follows that every such $n$ is finite.

The theorem below is a far reaching generalisation of Corollary 7 in [9] (and actually also Theorem 1 therein). It also shows that the existence of a relatively weakly compact $\alpha$-constrictor for a Markov semigroup leads to an interesting structure property of the fixed-point space of the dual semigroup.

Theorem 14. Assume that there exists a relatively weakly compact $\alpha$ constrictor $\mathcal{A} \subset \mathcal{M}_{*}$ for the Markov semigroup $\left(T_{t}: t \in G\right)$, where $0 \leqslant$ $\alpha<1$, or that $\mathcal{A}$ is a relatively weakly compact weak 0 -constrictor. Then

(i) $\left(T_{t}\right)$ is mean ergodic,

(ii) the fixed-point space $\left(\mathrm{Fix} T^{*}\right)^{h}$ is a JW-algebra being a finite direct sum of $J W$-factors of type $\mathrm{I}_{n_{i}}$ with all the $n_{i}$ finite.

Moreover, if the $T_{t}^{*}$ are strongly positive, then the fixed-point spaces Fix $T^{*}$ and Fix $T$ are finite dimensional.

Proof: Let us begin with the following simple observation. Put

$$
\mathcal{A}^{ \pm}=\mathcal{A} \cup(-\mathcal{A}) .
$$

Then $\mathcal{A}^{ \pm}$is clearly relatively weakly compact. Let $\left(p_{n}\right)$ be an arbitrary sequence of projections in $\mathcal{M}$ decreasing to 0 . Since $\mathcal{A}^{ \pm}$is relatively weakly compact, $[\mathbf{3 2}$, Theorem III.5.4] yields that for any $\varepsilon>0$ there is $n_{0}$ such that for all $n \geqslant n_{0}$ and all $\psi \in \mathcal{A}^{ \pm}$we have

$$
\left|\psi\left(p_{n}\right)\right|<\varepsilon .
$$


It follows that for any convex combination $\sum_{i=1}^{m} \lambda_{i} \psi_{i} \in \operatorname{conv} \mathcal{A}^{ \pm}$, the inequality

$$
\left|\sum_{i=1}^{m} \lambda_{i} \psi_{i}\left(p_{n}\right)\right|<\varepsilon
$$

holds for $n \geqslant n_{0}$. Consequently, the theorem quoted above yields that $\operatorname{conv} \mathcal{A}^{ \pm}$is relatively weakly compact. Thus $\overline{\operatorname{conv} \mathcal{A}^{ \pm}}$is weakly compact. Moreover, it is immediately seen that

$$
\left(\overline{\operatorname{conv} \mathcal{A}^{ \pm}}\right)^{ \pm}=\overline{\operatorname{conv} \mathcal{A}^{ \pm}}
$$

Since $\overline{\operatorname{conv} \mathcal{A}^{ \pm}}$contains $\mathcal{A}$, it is also an $\alpha$-constrictor, so we may assume, taking $\overline{\operatorname{conv} \mathcal{A}^{ \pm}}$in place of $\mathcal{A}$, that $\mathcal{A}$ is a convex weakly compact $\alpha$-constrictor such that for any $\psi \in \mathcal{A}$ we have $-\psi \in \mathcal{A}$.

Assume first that $\mathcal{A}$ is an $\alpha$-constrictor.

We shall prove (i). Let $\varphi$ be an arbitrary normal state. Since $\mathcal{A}$ is an $\alpha$-constrictor, there are $\psi_{t} \in \mathcal{A}$ such that

$$
\limsup _{t \in G}\left\|T_{t} \varphi-\psi_{t}\right\| \leqslant \alpha .
$$

Take arbitrary $\beta$ such that $\alpha<\beta<1$. Then there is $t_{0} \in G$ such that for all $t \geqslant t_{0}$ we have

$$
\left\|T_{t} \varphi-\psi_{t}\right\| \leqslant \beta
$$

Denoting

$$
\varphi_{t}= \begin{cases}T_{t} \varphi & \text { if } t \geqslant t_{0} \\ 0 & \text { otherwise }\end{cases}
$$

and

$$
\psi_{t}^{\prime}= \begin{cases}\psi_{t} & \text { if } t \geqslant t_{0} \\ 0 & \text { otherwise }\end{cases}
$$

we obtain

$$
\left\|\varphi_{t}-\psi_{t}^{\prime}\right\| \leqslant \beta \quad \text { for all } t \in G .
$$

Let $m$ be an invariant mean on $\ell^{\infty}(G)$. Then

$$
\left\|\tilde{m}_{\left[\varphi_{t}\right]}-\tilde{m}_{\left[\psi_{t}^{\prime}\right]}\right\| \leqslant \beta .
$$

We have

and

$$
\widetilde{m}_{\left[\varphi_{t}\right]}=\widetilde{m}_{\left[T_{t} \varphi\right]}=m_{T}(\varphi),
$$

$$
\widetilde{m}_{\left[\psi_{t}^{\prime}\right]}=\widetilde{m}_{\left[\psi_{t}\right]} \cdot
$$

Thus, putting $\widetilde{\psi}=\widetilde{m}_{\left[\psi_{t}\right]}$, we obtain

$$
\left\|m_{T}(\varphi)-\widetilde{\psi}\right\| \leqslant \beta .
$$


Moreover, formula (7) yields

$$
\widetilde{\psi}=\widetilde{m}_{\left[\psi_{t}\right]}=\lim _{\gamma} \sum_{i=1}^{n_{\gamma}} \lambda_{i}^{\gamma} \psi_{t_{i}^{\gamma}},
$$

and from the assumption of the convexity and weak compactness of $\mathcal{A}$ we obtain

$$
\widetilde{\psi}=\widetilde{m}_{\left[\psi_{t}\right]} \in \mathcal{A} .
$$

For arbitrary $\varphi \in \mathcal{M}_{*}^{+}$, formula (25) yields

$$
\left\|m_{T}(\varphi)-(\|\varphi\| \widetilde{\psi})\right\| \leqslant \beta\|\varphi\|
$$

for some $\tilde{\psi} \in \mathcal{A}$. For arbitrary $\varphi \in \mathcal{M}_{*}^{h}$, we have the Jordan decomposition $\varphi=\varphi^{+}-\varphi^{-}$. Consequently, there are $\widetilde{\psi}^{+}, \widetilde{\psi}^{-} \in \mathcal{A}$ such that

$$
\left\|m_{T}\left(\varphi^{+}\right)-\left(\left\|\varphi^{+}\right\| \widetilde{\psi}^{+}\right)\right\| \leqslant \beta\left\|\varphi^{+}\right\|
$$

and

$$
\left\|m_{T}\left(\varphi^{-}\right)-\left(\left\|\varphi^{-}\right\| \widetilde{\psi}^{-}\right)\right\| \leqslant \beta\left\|\varphi^{-}\right\|
$$

which leads to the inequality

$$
\begin{aligned}
\left\|m_{T}(\varphi)-\left(\left\|\varphi^{+}\right\| \widetilde{\psi}^{+}-\left\|\varphi^{-}\right\| \widetilde{\psi}^{-}\right)\right\| & \leqslant \beta\left(\left\|\varphi^{+}\right\|+\left\|\varphi^{-}\right\|\right) \\
& =\beta\|\varphi\| .
\end{aligned}
$$

Denote

$$
\psi=\left\|\varphi^{+}\right\| \widetilde{\psi}^{+}-\left\|\varphi^{-}\right\| \widetilde{\psi}^{-} .
$$

Then $\psi \in \mathcal{M}_{*}$. We have, for all $\varphi \in \mathcal{M}_{*}^{h}$, and the decomposition $m_{T}(\varphi)=$ $m_{T}(\varphi)_{n}+m_{T}(\varphi)_{s}$

$$
\beta\|\varphi\| \geqslant\left\|m_{T}(\varphi)-\psi\right\| \geqslant\left\|R^{\perp}\left(m_{T}(\varphi)-\psi\right)\right\|=\left\|m_{T}(\varphi)_{s}\right\| .
$$

Let $\varphi \in \mathcal{M}_{*}$. For arbitrary $x \in \operatorname{Fix} T^{*}$, we have

$$
\begin{aligned}
m_{T}(\varphi)(x) & =\widetilde{m}_{\left[T_{t} \varphi\right]}(x)=m\left(\left[t \mapsto\left(T_{t} \varphi\right)(x)\right]\right) \\
& =m\left(\left[t \mapsto \varphi\left(T_{t}^{*} x\right)\right]\right)=m([t \mapsto \varphi(x)])=\varphi(x) .
\end{aligned}
$$

Now we shall show that Fix $T$ separates the points of Fix $T^{*}$. To this end, it is enough to show that for any $x=x^{*} \in \operatorname{Fix} T^{*}$ with $\|x\|=1$ there is $\varphi \in \operatorname{Fix} T$ such that $\varphi(x) \neq 0$. Choose $\varepsilon>0$ such that $\beta+\varepsilon<1$. There is $\varphi \in \mathcal{M}_{*}^{h}$ such that $\|\varphi\|=1$ and

$$
|\varphi(x)|>1-\varepsilon \text {. }
$$


Let $\mathbb{E}$ be the quasi-ergodic projection associated with $m$. Taking into account the decomposition (17), we get

$$
\begin{aligned}
1-\varepsilon<|\varphi(x)| & =\left|m_{T}(\varphi)(x)\right| \leqslant|(\varphi \circ \mathbb{E})(x)|+\left|m_{T}(\varphi)_{s}(x)\right| \\
& \leqslant|(\varphi \circ \mathbb{E})(x)|+\beta,
\end{aligned}
$$

which yields

$$
|(\varphi \circ \mathbb{E})(x)|>1-(\beta+\varepsilon)>0 .
$$

Since $\varphi \circ \mathbb{E} \in \operatorname{Fix} T$, we have the desired separation property. From $[\mathbf{2 4}$, Lemma 14], it follows that $\mathbb{E}(\mathcal{M})=$ Fix $T^{*}$. Thus, by virtue of Proposition 3 we obtain (i).

Now we prove (ii). Let $\mathbb{E}^{T}$ be the ergodic projection. For its preadjoint $\mathbb{E}_{*}^{T}: \mathcal{M}_{*} \rightarrow \mathcal{M}_{*}$ defined as

$$
\mathbb{E}_{*}^{T} \varphi=\varphi \circ \mathbb{E}^{T}, \quad \varphi \in \mathcal{M}_{*},
$$

we have that $\mathbb{E}_{*}^{T}$ is $\sigma$-weakly continuous. Thus $\mathbb{E}_{*}^{T}(\mathcal{A})$ is $\sigma$-weakly compact. Clearly, $\mathbb{E}_{*}^{T}(\mathcal{A})=\mathbb{E}_{*}^{T}(\mathcal{A})^{ \pm}$. First we shall show that for the unit ball $(\text { Fix } T)_{1}^{h}$ we have the inclusion

$$
(\operatorname{Fix} T)_{1}^{h} \subset \frac{1}{1-\alpha} \mathbb{E}_{*}^{T}(\mathcal{A}) .
$$

Take an arbitrary $\varphi \in(\operatorname{Fix} T)^{h},\|\varphi\| \leqslant 1$, and let $\psi$ be defined by formula (27). Then $\psi \in \mathcal{A}$ and, according to formula (26), we get for each $1>\beta>\alpha$

$$
\|\varphi-(\|\varphi\| \psi)\| \leqslant \beta\|\varphi\| .
$$

Since $\mathbb{E}_{*}^{T} \varphi=\varphi$, this gives

$$
\left\|\varphi-\left(\|\varphi\| \mathbb{E}_{*}^{T} \psi\right)\right\| \leqslant \beta\|\varphi\| \text {. }
$$

Denoting

$$
\rho=\mathbb{E}_{*}^{T} \psi,
$$

we get the following condition: for any $\varphi \in(\operatorname{Fix} T)^{h},\|\varphi\| \leqslant 1$, there is $\rho \in \mathbb{E}_{*}^{T}(\mathcal{A})$ such that

$$
\|\varphi-(\|\varphi\| \rho)\| \leqslant \beta\|\varphi\| \text {. }
$$

Put

$$
\rho_{1}=\|\varphi\| \rho \text {. }
$$

Then $\rho_{1} \in \mathbb{E}_{*}^{T}(\mathcal{A}), \varphi-\rho_{1} \in(\operatorname{Fix} T)^{h}$, and $\left\|\varphi-\rho_{1}\right\| \leqslant \beta\|\varphi\| \leqslant \beta$. For the functional $\varphi-\rho_{1} \in(\text { Fix } T)^{h}$, we obtain on account of condition (28) that there is $\rho_{2}^{\prime} \in \mathbb{E}_{*}^{T}(\mathcal{A})$ such that

$$
\left\|\left(\varphi-\rho_{1}\right)-\left(\left\|\varphi-\rho_{1}\right\| \rho_{2}^{\prime}\right)\right\| \leqslant \beta\left\|\varphi-\rho_{1}\right\| \leqslant \beta^{2} .
$$


Denoting

we obtain $\rho_{2} \in \beta \mathbb{E}_{*}^{T}(\mathcal{A})$ and

$$
\rho_{2}=\left\|\varphi-\rho_{1}\right\| \rho_{2}^{\prime},
$$

$$
\left\|\varphi-\left(\rho_{1}+\rho_{2}\right)\right\| \leqslant \beta^{2} .
$$

We proceed further by induction. Having chosen the elements $\rho_{1}, \ldots, \rho_{n}$ such that $\rho_{k} \in \beta^{k-1} \mathbb{E}_{*}^{T}(\mathcal{A}), k=1, \ldots, n$, and

$$
\left\|\varphi-\left(\rho_{1}+\cdots+\rho_{n}\right)\right\| \leqslant \beta^{n},
$$

we find $\rho_{n+1}^{\prime} \in \mathbb{E}_{*}^{T}(\mathcal{A})$ such that

$$
\begin{aligned}
\|\left(\varphi-\left(\rho_{1}+\cdots+\rho_{n}\right)\right)-(\| \varphi- & \left.\left(\rho_{1}+\cdots+\rho_{n}\right) \| \rho_{n+1}^{\prime}\right) \| \\
& \leqslant \beta^{n}\left\|\varphi-\left(\rho_{1}+\cdots+\rho_{n}\right)\right\| \leqslant \beta^{n+1},
\end{aligned}
$$

and putting

$$
\rho_{n+1}=\left\|\varphi-\left(\rho_{1}+\cdots+\rho_{n}\right)\right\| \rho_{n+1}^{\prime},
$$

we get $\rho_{n+1} \in \beta^{n} \mathbb{E}_{*}^{T}(\mathcal{A})$ and

$$
\left\|\varphi-\left(\rho_{1}+\cdots+\rho_{n+1}\right)\right\| \leqslant \beta^{n+1} .
$$

It follows that

$$
\lim _{n \rightarrow \infty}\left(\rho_{1}+\cdots+\rho_{n}\right)=\varphi,
$$

and since $\rho_{k} \in \beta^{k-1} \mathbb{E}_{*}^{T}(\mathcal{A})$, we have

$$
\rho_{1}+\cdots+\rho_{n} \in\left(1+\cdots+\beta^{n-1}\right) \mathbb{E}_{*}^{T}(\mathcal{A}) \subset \frac{1}{1-\beta} \mathbb{E}_{*}^{T}(\mathcal{A}) .
$$

From the weak compactness of the set $\frac{1}{1-\beta} \mathbb{E}_{*}^{T}(\mathcal{A})$, we obtain $\varphi \in \frac{1}{1-\beta} \mathbb{E}_{*}^{T}(\mathcal{A})$, which means that

$$
(\operatorname{Fix} T)_{1}^{h} \subset \frac{1}{1-\beta} \mathbb{E}_{*}^{T}(\mathcal{A})
$$

Consequently,

$$
(\operatorname{Fix} T)_{1}^{h} \subset \bigcap_{\beta>\alpha} \frac{1}{1-\beta} \mathbb{E}_{*}^{T}(\mathcal{A})=\frac{1}{1-\alpha} \mathbb{E}_{*}^{T}(\mathcal{A}),
$$

proving the claim. As a consequence of the inclusion above and the fact that $(\mathrm{Fix} T)_{1}^{h}$ is weakly closed, it follows that $(\mathrm{Fix} T)_{1}^{h}$ is weakly compact, hence $(\operatorname{Fix} T)_{1}$ is weakly compact. On account of [29, Exercise 4.1], we infer that $\operatorname{Fix} T$ is reflexive. It follows that $(\operatorname{Fix} T)^{*}$ is reflexive too, and since by Lemma $12(\operatorname{Fix} T)^{*}$ is isometrically isomorphic to $\mathbb{E}^{T}(\mathcal{M})=$ Fix $T^{*}$, we infer that $\operatorname{Fix} T^{*}$ is reflexive.

Let $\left\{e_{i}: i \in I\right\}$ be an arbitrary resolution of identity in $\operatorname{Fix} T^{*}$, i.e. $e_{i}$ are projections which sum up to $\mathbb{1}$, and let $\pi$ be an isometric Jordan 
representation of $\left(\left(\operatorname{Fix} T^{*}\right)^{h}, \bullet\right)$. Then, letting $\widehat{\pi}$ denote the induced Jordan representation of $\left(\operatorname{Fix} T^{*}, \bullet\right)$, we have that $\widehat{\pi}\left(e_{i}\right)$ is a resolution of identity in $\widehat{\pi}\left(\operatorname{Fix} T^{*}\right)$. Put

$$
\mathfrak{R}=\overline{\operatorname{Lin}\left\{\widehat{\pi}\left(e_{i}\right): i \in I\right\}},
$$

where the closure is in the norm topology. $\mathfrak{R}$ is a $\mathrm{C}^{*}$-algebra, and since $\widehat{\pi}\left(\right.$ Fix $\left.T^{*}\right)$ is reflexive, $\mathfrak{R}$ as a Banach subspace of $\widehat{\pi}\left(\operatorname{Fix} T^{*}\right)$ is reflexive too. But a $\mathrm{C}^{*}$-algebra is reflexive if and only if it is finite dimensional, which implies that $I$ is a finite set. Consequently, each resolution of identity in Fix $T^{*}$ is finite. Now the assertion follows from Proposition 13.

Assume now that the $T_{t}^{*}$ are strongly positive. From part (ii) we know that $\operatorname{Fix} T^{*}$ is reflexive. Since for the maps $T_{t}^{*}$ being strongly positive, Fix $T^{*}=\mathbb{E}^{T}(\mathcal{M})$ is a $\mathrm{W}^{*}$-algebra, its reflexivity yields the finiteness of its dimension. Since Fix $T^{*}=(\operatorname{Fix} T)^{*}$, we have $\operatorname{dim} \operatorname{Fix} T=\operatorname{dim} \operatorname{Fix} T^{*}$, and the conclusion follows.

Let now $\mathcal{A}$ be a weak 0 -constrictor. Then for each normal state $\varphi$, there are $\psi_{t} \in \mathcal{A}$ such that for each $x \in \mathcal{M}$

$$
\lim _{t \in G}\left(T_{t} \varphi-\psi_{t}\right)(x)=0 .
$$

Let $m$ be an invariant mean on $\ell^{\infty}(G)$. Then, for the function $t \mapsto$ $\left(T_{t} \varphi-\psi_{t}\right)(x)$, we have on account of relation $(3)$

$$
\begin{aligned}
0 & =m\left(\left[t \mapsto\left(T_{t} \varphi\right)(x)\right]\right)-m\left(\left[t \mapsto \psi_{t}(x)\right]\right) \\
& =m_{T}(\varphi)(x)-\widetilde{m}_{\left[\psi_{t}\right]}(x) .
\end{aligned}
$$

Since $\mathcal{A}$ is weakly compact and convex, we have $\widetilde{m}_{\left[\psi_{t}\right]} \in \mathcal{A}$, and thus $m_{T}(\varphi)=\widetilde{m}_{\left[\psi_{t}\right]} \in \mathcal{A}$ which means that $m_{T}(\varphi)$ is a normal state. Since normal states span the whole of $\mathcal{M}_{*}$, we obtain that $m_{T}$ sends normal functionals to normal ones, i.e. the semigroup $\left(T_{t}\right)$ is mean ergodic.

Assume now that $\varphi$ is an invariant state. Then

$$
\varphi-\psi_{t}=T_{t} \varphi-\psi_{t} \underset{t \in G}{\longrightarrow} 0 \quad \text { weakly, }
$$

which means that $\varphi \in \mathcal{A}$. For arbitrary $\varphi \in \mathcal{M}_{*}^{+}$with $\|\varphi\| \leqslant 1$, we have $\varphi \in\|\varphi\| \mathcal{A} \subset \mathcal{A}$. Let $\varphi \in(\text { Fix } T)^{h}$. For the Jordan decomposition $\varphi=$ $\varphi^{+}-\varphi^{-}$, we have

$$
\begin{aligned}
\varphi & =\varphi^{+}-\varphi^{-}=T_{t} \varphi=T_{t} \varphi^{+}-T_{t} \varphi^{-}, \\
\left\|T_{t} \varphi^{+}\right\| & =\left(T_{t} \varphi^{+}\right)(\mathbb{1})=\varphi^{+}(\mathbb{1})=\left\|\varphi^{+}\right\|, \quad \text { and } \\
\left\|T_{t} \varphi^{-}\right\| & =\left(T_{t} \varphi^{-}\right)(\mathbb{1})=\varphi^{-}(\mathbb{1})=\left\|\varphi^{-}\right\| .
\end{aligned}
$$

Thus, from the uniqueness of the decomposition, we obtain

$$
T_{t} \varphi^{+}=\varphi^{+}, \quad T_{t} \varphi^{-}=\varphi^{-},
$$


i.e. $\varphi^{+}, \varphi^{-} \in \operatorname{Fix} T$. For any $\varphi \in(\operatorname{Fix} T)^{h}$ with $\|\varphi\| \leqslant 1$, we have from the above that $\varphi^{+}, \varphi^{-} \in \operatorname{Fix} T$, and thus $\varphi^{+}, \varphi^{-} \in \mathcal{A}$. In particular, from the properties of $\mathcal{A}$ we obtain $-\varphi^{-} \in \mathcal{A}$. Consequently,

$$
\frac{1}{2} \varphi=\frac{1}{2} \varphi^{+}+\frac{1}{2}\left(-\varphi^{-}\right) \in \mathcal{A},
$$

which means that $(\operatorname{Fix} T)_{1}^{h} \subset 2 \mathcal{A}$, i.e. $(\operatorname{Fix} T)_{1}^{h}$ is weakly compact. The rest of the proof follows as in the first part.

Remark 5. The theorem above shows the advantage of using the abstract approach based on invariant means. Namely, if $\mathcal{A}$ is an $\alpha$-constrictor for the semigroup $\left(T_{t}\right)$, then there are $\psi_{t} \in \mathcal{A}$ and $\beta$ such that $\alpha<\beta<1$ and

$$
\left\|T_{t} \varphi-\psi_{t}\right\| \leqslant \beta
$$

for all sufficiently large $t$. However, to obtain a relation of this type for the Cesàro means

$$
A_{t}(\varphi)=\frac{1}{t} \int_{0}^{t} T_{s} \varphi d s,
$$

which is crucial in [9, Corollary 7$]$, where the inequality

$$
\left\|A_{t}(\varphi)-\rho_{t}\right\| \leqslant \beta
$$

is needed, requires some construction. First, the measurability of the function $t \mapsto \psi_{t}$ should be taken care of. This can be achieved by taking this function as a step function in such a way that relation (29) holds (possibly with some $\beta^{\prime}<1$ ). Then, defining

$$
\rho_{t}=\frac{1}{t} \int_{0}^{t} \psi_{s} d s
$$

one obtains the result.

If the constrictor is 'sufficiently small', then we get more. Recall that the semigroup $\left(T_{t}: t \in G\right)$ is said to be asymptotically stable if there exists a normal state $\psi$ such that for every normal state $\varphi$ we have

$$
\lim _{t \in G}\left\|T_{t} \varphi-\psi\right\|=0 .
$$

When it exists, such a state $\psi$ is clearly $\left(T_{t}\right)$-invariant and unique. The result that follows shows asymptotic stability of the semigroup under an assumption on the diameter of the constrictor, and seems to be new even in the classical case. Let $\operatorname{diam} \mathcal{A}$ be the diameter of $\mathcal{A}$ defined as

$$
\operatorname{diam} \mathcal{A}=\sup \{\|\varphi-\psi\|: \varphi, \psi \in \mathcal{A}\} .
$$


Theorem 15. Assume that there exists a relatively weakly compact $\alpha$ constrictor $\mathcal{A} \subset \mathcal{M}_{*}$ for the Markov semigroup $\left(T_{t}: t \in G\right)$, where $0 \leqslant$ $\alpha<1$ and $\operatorname{diam} \mathcal{A} \leqslant 2(1-\alpha)$. Then $\left(T_{t}\right)$ is asymptotically stable.

Proof: Take $\alpha<\beta<1$ such that $\operatorname{diam} \mathcal{A}<2(1-\beta)$. Thus, denoting $\gamma=\operatorname{diam} \mathcal{A}$, we have

$$
\beta+\frac{\gamma}{2}<1
$$

Theorem 14 implies that $\left(T_{t}\right)$ is mean ergodic, hence there is an invariant normal state $\rho$ (we can simply take $\rho=\varphi \circ \mathbb{E}^{T}$ where $\varphi$ is a normal state and $\mathbb{E}^{T}$ is the ergodic projection). For any normal state $\varphi$, we have $\left\|T_{t} \varphi-\rho\right\|=\left\|T_{t}(\varphi-\rho)\right\|$, and since $(\varphi-\rho)(\mathbb{1})=0$, in order to prove that $\left\|T_{t} \varphi-\rho\right\| \rightarrow 0$ it is enough to show that for any $\omega \in \mathcal{M}_{*}^{h}$ such that $\omega(\mathbb{1})=0$, we have $\left\|T_{t} \omega\right\| \rightarrow 0$. The net $\left\{\left\|T_{t} \omega\right\|: t \in G\right\}$ is non-increasing, so assume that $\left\|T_{t} \omega\right\| \rightarrow \eta$ for some $\eta>0$. Take an arbitrary $\varepsilon>0$ and choose $t_{0}$ such that

$$
\left\|T_{t_{0}} \omega\right\|<\eta+\varepsilon
$$

Denoting $\omega_{0}=T_{t_{0}} \omega$, we have $\left\|\omega_{0}\right\|<\eta+\varepsilon,\left\|T_{t} \omega_{0}\right\| \rightarrow \eta$, and

$$
\omega_{0}(\mathbb{1})=\left(T_{t_{0}} \omega\right)(\mathbb{1})=\omega\left(T_{t_{0}}^{*}(\mathbb{1})\right)=\omega(\mathbb{1})=0 .
$$

For the Jordan decomposition $\omega_{0}=\omega_{0}^{+}-\omega_{0}^{-}$, we obtain

$$
0=\omega_{0}(\mathbb{1})=\omega_{0}^{+}(\mathbb{1})-\omega_{0}^{-}(\mathbb{1})=\left\|\omega_{0}^{+}\right\|-\left\|\omega_{0}^{-}\right\|,
$$

and since $\left\|\omega_{0}\right\|=\left\|\omega_{0}^{+}\right\|+\left\|\omega_{0}^{-}\right\|$, we get $\left\|\omega_{0}^{+}\right\|=\left\|\omega_{0}^{-}\right\|=\frac{1}{2}\left\|\omega_{0}\right\|$. Since $\mathcal{A}$ is an $\alpha$-constrictor, condition (9) yields that there are $\varphi_{t}, \psi_{t} \in \mathcal{A}$ such that

$$
\begin{aligned}
& \left\|T_{t} \omega_{0}^{+}-\left(\left\|\omega_{0}^{+}\right\| \varphi_{t}\right)\right\|<\beta\left\|\omega_{0}^{+}\right\|=\frac{1}{2} \beta\left\|\omega_{0}\right\| \text { and } \\
& \left\|T_{t} \omega_{0}^{-}-\left(\left\|\omega_{0}^{-}\right\| \psi_{t}\right)\right\|<\beta\left\|\omega_{0}^{-}\right\|=\frac{1}{2} \beta\left\|\omega_{0}\right\|,
\end{aligned}
$$

for sufficiently large $t$. Thus we obtain

$$
\begin{aligned}
\left\|T_{t} \omega_{0}\right\| \leqslant & \left\|T_{t} \omega_{0}^{+}-\left(\left\|\omega_{0}^{+}\right\| \varphi_{t}\right)\right\|+\left\|\left(\left\|\omega_{0}^{+}\right\| \varphi_{t}\right)-\left(\left\|\omega_{0}^{-}\right\| \psi_{t}\right)\right\| \\
& +\left\|T_{t} \omega_{0}^{-}-\left(\left\|\omega_{0}^{-}\right\| \psi_{t}\right)\right\| \\
\leqslant & \frac{1}{2} \beta\left\|\omega_{0}\right\|+\frac{1}{2}\left\|\omega_{0}\right\|\left\|\varphi_{t}-\psi_{t}\right\|+\frac{1}{2} \beta\left\|\omega_{0}\right\| \\
\leqslant & \left(\beta+\frac{\gamma}{2}\right)\left\|\omega_{0}\right\|<\left(\beta+\frac{\gamma}{2}\right)(\eta+\varepsilon) .
\end{aligned}
$$


Since $\left\|T_{t} \omega_{0}\right\| \rightarrow \eta$ we obtain, passing to the limit in the inequality above, that $\eta \leqslant\left(\beta+\frac{\gamma}{2}\right)(\eta+\varepsilon)$, and since $\varepsilon$ was arbitrary, we get $\eta \leqslant\left(\beta+\frac{\gamma}{2}\right) \eta$, i.e. $1 \leqslant \beta+\frac{\gamma}{2}-$ a contradiction.

Observe that, as a simple corollary to the theorem above we obtain the noncommutative counterpart of a known classical fact - point (i) below in Corollary 16 (a much deeper result, however, only for a strongly continuous semigroup $\left(T_{t}: t \geqslant 0\right)$ or the semigroup $\left(T^{n}: n=0,1, \ldots\right)$ will be shown later in Theorem 19), and a general version of [9, Theorem 2] and [6, Theorem 6] - point (ii) below in Corollary 16. It is worth observing that in $\left[\mathbf{9}\right.$, Theorem 2] a strongly continuous semigroup $\left(T_{t}: t \geqslant 0\right)$ acting on the predual of a von Neumann algebra is considered, while [6, Theorem 6] deals with an abelian semigroup $\left(T_{t}: t \in G\right)$ acting on an $L^{1}$-space, and it is shown that a condition like the one in point (ii) below yields that the net $\left\{T_{t}: t \in G\right\}$ is a so-called Lotz-Räbiger net which means that for arbitrary $\varphi \in \mathcal{M}_{*}$ and $s \in G$, we have

$$
\lim _{t \in G}\left(T_{t+s}-T_{t}\right) \varphi=0 .
$$

Then the conclusion follows from a corresponding result for Lotz-Räbiger nets.

Corollary 16. Assume that there is a normal positive linear functional $\psi$ on $\mathcal{M}$ such that

(i) $\|\psi\|<2$, and for each normal state $\varphi$ on $\mathcal{M}$

or

$$
\lim _{t \in G}\left\|\left(T_{t} \varphi-\psi\right)^{+}\right\|=0
$$

(ii) for each normal state $\varphi$ on $\mathcal{M}$

$$
\lim _{t \in G}\left\|\left(T_{t} \varphi-\psi\right)^{-}\right\|=0 .
$$

Then $\left(T_{t}\right)$ is asymptotically stable.

Proof: Indeed, for any $\omega \in \mathcal{M}_{*}^{h}$ and its Jordan decomposition $\omega=\omega^{+}{ }_{-}$ $\omega^{-}$, we have

$$
\|\omega\|=\left\|\omega^{+}\right\|+\left\|\omega^{-}\right\| \quad \text { and } \quad \omega(\mathbb{1})=\left\|\omega^{+}\right\|-\left\|\omega^{-}\right\|,
$$

so the formula

$$
\|\omega\|=2\left\|\omega^{+}\right\|-\omega(\mathbb{1})=2\left\|\omega^{-}\right\|+\omega(\mathbb{1})
$$

holds. Thus

$$
\begin{aligned}
\left\|T_{t} \varphi-\psi\right\| & =2\left\|\left(T_{t} \varphi-\psi\right)^{+}\right\|-\left(T_{t} \varphi-\psi\right)(\mathbb{1}) \\
& =2\left\|\left(T_{t} \varphi-\psi\right)^{+}\right\|+\|\psi\|-1 \\
& =2\left\|\left(T_{t} \varphi-\psi\right)^{-}\right\|+1-\|\psi\| .
\end{aligned}
$$


Passing to the limit we obtain, for $\alpha=\|\psi\|-1<1$ in case (i) or $\alpha=1-\|\psi\|<1$ in case (ii),

$$
\lim _{t \in G}\left\|T_{t} \varphi-\psi\right\|=\alpha .
$$

Thus Theorem 15 gives the claim upon taking $\{\psi\}$ as the constrictor.

Remark 6. Condition (ii) in the above corollary was described in [9] as the existence of a lower-bound for the semigroup $\left(T_{t}\right)$. Analogously, condition (i) could be named as the existence of an upper-bound for $\left(T_{t}\right)$ (as a matter of fact, this kind of definition is used in [18, Section 1, p. 37] for a Markov operator on $L^{1}$ ). As is seen, the existence of a lowerbound implies much more than the existence of an upper-bound. In particular, the fixed-point space for an arbitrary semigroup with a lowerbound is one-dimensional while the fixed-point space for the strongly continuous semigroup $\left(T_{t}: t \geqslant 0\right)$ (or $\left(T^{n}: n=0,1, \ldots\right)$ ) with an upper-bound, described in Theorem 19, need not be so simple. It is interesting that a similar situation holds for mean boundedness which is shown in Theorems 20 and 22.

In general, the corollary above as well as Theorems 19, 20, and 22 that follow deal, at least partly, with asymptotic properties of the semigroup $\left(T_{t}: t \in G\right)$, being at the same time examples of lower bound (or upper bound) theorems. Investigations of this type were performed, for example, in a more general setting of ordered Banach spaces with generating cone in [12], where almost periodicity and mean ergodicity are related with asymptotic domination. In a similar setting of base norm spaces, uniform asymptotic stability, and mean ergodicity, where the appropriate limits are taken in the operator norm, were investigated in $[\mathbf{1 0}]$.

Lemma 17. Let $\widetilde{\varphi}$ be a state on $\mathcal{M}$ majorized by a normal positive linear functional $\psi$. Then $\widetilde{\varphi}$ is normal.

Proof: From the inequality

$$
0 \leqslant \widetilde{\varphi} \leqslant \psi
$$

we get for the projection $R^{\perp}$

$$
0 \leqslant R^{\perp} \widetilde{\varphi} \leqslant R^{\perp} \psi=0,
$$

which yields $R^{\perp} \widetilde{\varphi}=0$. Thus $\widetilde{\varphi}=R \widetilde{\varphi}$, showing the claim.

The next result is well-known in the theory of von Neumann algebras. For the sake of completeness, we present its proof here. 
Lemma 18. Let $\mathcal{M}$ be a von Neumann algebra, and let $e_{1}, \ldots, e_{n}$ be minimal projections in $\mathcal{M}$ such that $\sum_{i=1}^{n} e_{i}=\mathbb{1}$. Then $\operatorname{dim} \mathcal{M} \leqslant n^{2}$.

Proof: If $\mathcal{M}$ is a factor, then its type is $\mathrm{I}_{n}$, so $\mathcal{M}$ is ${ }^{*}$-isomorphic to $\mathbb{B}\left(\mathbb{C}^{n}\right)$ whose dimension equals $n^{2}$.

Let now $\mathcal{M}$ be arbitrary. For each central projection $z$ of $\mathcal{M}$ and each $e_{i}$, the minimality of $e_{i}$ yields $e_{i} z=e_{i}$ or 0 . Consequently, $z$ is a sum of some $e_{i}$. Taking all possible products of (finitely many) central projections we get minimal central projections $z_{1}, \ldots, z_{m}$ with sum $\mathbb{1}$, each being a sum of some $e_{i}$ :

$$
z_{k}=\sum_{r=1}^{n_{k}} e_{i_{r}^{(k)}}
$$

Moreover,

$$
n_{1}+\cdots+n_{m}=n
$$

We have

$$
\mathcal{M}=\mathcal{M}_{z_{1}} \oplus \cdots \oplus \mathcal{M}_{z_{m}},
$$

and since $z_{k}$ are minimal in the centre of $\mathcal{M}$, all $\mathcal{M}_{z_{k}}$ are factors of type $\mathrm{I}_{n_{k}}$. Hence

$$
\operatorname{dim} \mathcal{M}_{z_{k}}=n_{k}^{2}
$$

and

$$
\operatorname{dim} \mathcal{M}=\operatorname{dim} \mathcal{M}_{z_{1}}+\cdots+\operatorname{dim} \mathcal{M}_{z_{m}}=n_{1}^{2}+\cdots+n_{m}^{2} \leqslant n^{2} .
$$

Before proceeding further, let us explain the situation which occurs in the next theorem. The existence of an upper-bound for a semigroup $\left(T_{t}\right.$ : $t \in G)$ implies some regularity of the limit points 'at infinity' of $\left(T_{t} \varphi\right.$ : $t \in G)$. However, in general, nothing can be said about other limit points. This is why we restrict attention to more specific semigroups such as $[0,+\infty)$ or $\mathbb{Z}_{+}$together with the natural assumption of strong continuity, which means that for every $\varphi \in \mathcal{M}_{*}$ the map $t \mapsto T_{t} \varphi$ is (norm- or weakly-) continuous. This assumption allows us to obtain the property of ultraweak almost periodicity for the semigroup $\left\{T_{t}^{*}: t \in G\right\}$ which is basic for the conclusions. It should be mentioned that this is the only place where we deal with the concrete strongly continuous semigroups $\left(T_{t}: t \geqslant 0\right)$ or $\left(T^{n}: n=0,1, \ldots\right)$. 
Theorem 19. Let $G=[0,+\infty)$ or $G=\mathbb{Z}_{+}$, and for the case $G=$ $[0,+\infty)$, let $\left(T_{t}: t \geqslant 0\right)$ be strongly continuous. Assume that there is a normal positive linear functional $\psi$ such that for each normal state $\varphi$ we have

$$
\lim _{t \rightarrow \infty}\left\|\left(T_{t} \varphi-\psi\right)^{+}\right\|=0 .
$$

Then $\left(T_{t}\right)$ is mean ergodic, and the $J W$-algebra $(\text { Fix } T)^{h}$ is a finite direct sum of JW-factors of type $\mathrm{I}_{n_{i}}$ with all the $n_{i}$ finite. Moreover, if the $T_{t}^{*}$ are strongly positive, then $\operatorname{dim} \operatorname{Fix} T \leqslant\lfloor\|\psi\|\rfloor^{2}$, where $\lfloor\|\psi\|\rfloor$ stands for the integer part of $\|\psi\|$.

Proof: It is enough to consider only the case $G=[0,+\infty)$ since the proof for the discrete case is similar.

Recall that a semigroup $\mathfrak{S}$ of positive normal linear contractions on $\mathcal{M}$ is called ultraweakly almost periodic if it satisfies any of the following equivalent conditions:

(i) for each $\omega \in \mathcal{M}_{*},\{\omega \circ S: S \in \mathfrak{S}\}$ is relatively weakly compact,

(ii) the point- $\sigma$-weak closure of $\mathfrak{S}$ consists of normal operators,

(iii) $\mathfrak{S}$ is equicontinuous in the Mackey topology $\tau\left(\mathcal{M}, \mathcal{M}_{*}\right)$.

(See $[\mathbf{2 0}, \mathbf{2 3}, \mathbf{3 3}]$.) Consider the semigroup $\left\{T_{t}^{*}: t \geqslant 0\right\}$ of positive normal linear contractions on $\mathcal{M}$. Since this semigroup is strongly continuous, it follows that its point- $\sigma$-weak closure consists of all $T_{t}^{*}$ 's and all possible limits at infinity of the $T_{t}^{*}$ 's. In other words, $S$ belongs to the closure if either $S=T_{t_{0}}^{*}$ for some $t_{0}$ or there is a net $\left\{t_{\gamma}\right\}$ of nonnegative real numbers tending to infinity such that, for any $x \in \mathcal{M}$ and any $\varphi \in \mathcal{M}_{*}$,

$$
\varphi(S x)=\lim _{\gamma} \varphi\left(T_{t_{\gamma}}^{*} x\right)=\lim _{\gamma}\left(T_{t_{\gamma}} \varphi\right)(x)
$$

i.e.

$$
\text { weak }^{*}-\lim _{\gamma} T_{t_{\gamma}} \varphi=\varphi \circ S \text {. }
$$

(We consider the functionals $T_{t_{\gamma}} \varphi$ and $\varphi \circ S$ as elements of $\mathcal{M}^{*}$.) For each normal state $\varphi$, the following inequality holds

$$
T_{t_{\gamma}} \varphi \leqslant \psi+\left(T_{t_{\gamma}} \varphi-\psi\right)^{+} .
$$

Thus, passing to the weak ${ }^{*}$-limit, we obtain

$$
\varphi \circ S \leqslant \psi
$$


On account of Lemma 17, $\varphi \circ S$ is a normal state. Since each $\varphi \in \mathcal{M}_{*}$ is a linear combination of normal states, we get that for every $\varphi \in \mathcal{M}_{*}$ we have $\varphi \circ S \in \mathcal{M}_{*}$, which means that $S$ is a normal map. This shows that the point- $\sigma$-weak closure of $\left\{T_{t}^{*}: t \geqslant 0\right\}$ consists of normal operators. Thus the semigroup $\left\{T_{t}^{*}: t \geqslant 0\right\}$ is ultraweakly almost periodic. By virtue of [3, Chapter V.6.4], the convex hull of $\left\{T_{t}: t \geqslant 0\right\}$ is also ultraweakly almost periodic (alternatively, one may use [32, Theorem III.5.4]). Let $m$ be an invariant mean on $\ell^{\infty}([0,+\infty))$ and consider the map $m_{T} \mid \mathcal{M}_{*}: \mathcal{M}_{*} \rightarrow \mathcal{M}^{*}$. On account of (10), we have

$$
m_{T}(\varphi)(x)=\lim _{\gamma} \sum_{i=1}^{n_{\gamma}} \lambda_{i}^{\gamma} T_{t_{i}^{\gamma}} \varphi(x)=\lim _{\gamma} \varphi\left(\sum_{i=1}^{n_{\gamma}} \lambda_{i}^{\gamma} T_{t_{i}^{\gamma}}^{*} x\right), \quad x \in \mathcal{M} .
$$

Thus, for $\left(m_{T} \mid \mathcal{M}_{*}\right)^{*} \mid \mathcal{M}: \mathcal{M} \rightarrow \mathcal{M}$, we get

$$
\varphi\left(\left(m_{T} \mid \mathcal{M}_{*}\right)^{*}(x)\right)=m_{T}(\varphi)(x)=\lim _{\gamma} \varphi\left(\sum_{i=1}^{n_{\gamma}} \lambda_{i}^{\gamma} T_{t_{i}^{\gamma}}^{*} x\right),
$$

which means that $\left(m_{T} \mid \mathcal{M}_{*}\right)^{*} \mid \mathcal{M}$ is a limit in the point- $\sigma$-weak topology of convex combinations of elements from $\left\{T_{t}^{*}: t \geqslant 0\right\}$. The ultraweak almost periodicity of this set implies that $\left(m_{T} \mid \mathcal{M}_{*}\right)^{*} \mid \mathcal{M}$ is a normal map. Consequently, for each $\varphi \in \mathcal{M}_{*}$, we have

$$
\varphi \circ\left(m_{T} \mid \mathcal{M}_{*}\right)^{*} \mid \mathcal{M} \in \mathcal{M}_{*} .
$$

But clearly,

$$
\varphi \circ\left(m_{T} \mid \mathcal{M}_{*}\right)^{*} \mid \mathcal{M}=m_{T}(\varphi) .
$$

Thus $m_{T}$ sends the normal functionals to normal ones, i.e. $\left(T_{t}\right)$ is mean ergodic.

Observe that from the assumption we immediately obtain the relation

$$
\varphi \leqslant \psi
$$

for each invariant normal state $\varphi$.

Suppose that $\varphi$ is a normal invariant state and let $e$ be a projection in the JW-algebra $\left(\left(\operatorname{Fix} T^{*}\right)^{h}, \bullet\right)$. We aim at showing that the functional e $\varphi$ is invariant. As before, let $\mathbb{E}^{T}$ stand for the ergodic projection. For arbitrary $a, b \in \mathcal{M}$ we have

$$
a b a=2 a \circ(a \circ b)-a^{2} \circ b,
$$


and thus for any $x \in \mathcal{M}$ we get, by virtue of equality (20) and relation (21),

$$
\begin{aligned}
(e \varphi e)\left(\mathbb{E}^{T} x\right) & =\varphi\left(e\left(\mathbb{E}^{T} x\right) e\right)=2 \varphi\left(e \circ\left(e \circ \mathbb{E}^{T} x\right)\right)-\varphi\left(e^{2} \circ \mathbb{E}^{T} x\right) \\
& =2 \varphi\left(\mathbb{E}^{T}\left(e \circ\left(e \circ \mathbb{E}^{T} x\right)\right)\right)-\varphi\left(\mathbb{E}^{T}\left(e^{2} \circ \mathbb{E}^{T} x\right)\right) \\
& =2 \varphi\left(\mathbb{E}^{T}\left(e \circ \mathbb{E}^{T}\left(e \circ \mathbb{E}^{T} x\right)\right)\right)-\varphi\left(\mathbb{E}^{T}\left(\mathbb{E}^{T} e^{2} \circ \mathbb{E}^{T} x\right)\right) \\
& =2 \varphi\left(\mathbb{E}^{T}\left(e \circ \mathbb{E}^{T}(e \circ x)\right)\right)-\varphi\left(\mathbb{E}^{T}\left(e \circ \mathbb{E}^{T} x\right)\right) \\
& =2 \varphi\left(\mathbb{E}^{T}(e \circ(e \circ x))\right)-\varphi\left(\mathbb{E}^{T}(e \circ x)\right) \\
& =2 \varphi(e \circ(e \circ x))-\varphi(e \circ x) .
\end{aligned}
$$

Take an arbitrary positive linear functional $\psi$ on $\mathcal{M}$ and define a sesquilinear form $[\cdot, \cdot]_{\psi}$ on $\mathcal{M} \times \mathcal{M}$ by the formula

$$
[x, y]_{\psi}=2 \psi\left(\mathbb{E}^{T}\left(x^{*} \circ y\right)\right)-\psi\left(\mathbb{E}^{T}\left(x^{*} \circ \mathbb{E}^{T} y\right)\right)-\psi\left(\mathbb{E}^{T}\left(\mathbb{E}^{T} x^{*} \circ y\right)\right) .
$$

We have

$x^{*} \circ x-x^{*} \circ \mathbb{E}^{T} x-\mathbb{E}^{T} x^{*} \circ x+\mathbb{E}^{T} x^{*} \circ \mathbb{E}^{T} x=\left(x-\mathbb{E}^{T} x\right)^{*} \circ\left(x-\mathbb{E}^{T} x\right) \geqslant 0$.

Thus

$$
\begin{aligned}
0 & \leqslant \mathbb{E}^{T}\left(x^{*} \circ x\right)-\mathbb{E}^{T}\left(x^{*} \circ \mathbb{E}^{T} x\right)-\mathbb{E}^{T}\left(\mathbb{E}^{T} x^{*} \circ x\right)+\mathbb{E}^{T}\left(\mathbb{E}^{T} x^{*} \circ \mathbb{E}^{T} x\right) \\
& \leqslant 2 \mathbb{E}^{T}\left(x^{*} \circ x\right)-\mathbb{E}^{T}\left(x^{*} \circ \mathbb{E}^{T} x\right)-\mathbb{E}^{T}\left(\mathbb{E}^{T} x^{*} \circ x\right),
\end{aligned}
$$

so $[\cdot, \cdot]_{\psi}$ is positive. For an arbitrary projection $e$ in $\left(\left(\operatorname{Fix} T^{*}\right)^{h}, \bullet\right)($ i.e. $e=$ $e \bullet e$ ) and $x \in \mathcal{M}$, we have

$$
\begin{aligned}
{\left[x^{*}, e^{2}\right]_{\psi} } & =2 \psi\left(\mathbb{E}^{T}\left(x \circ e^{2}\right)\right)-\psi\left(\mathbb{E}^{T}\left(x \circ \mathbb{E}^{T} e^{2}\right)-\psi\left(\mathbb{E}^{T}\left(\left(\mathbb{E}^{T} x\right) \circ e^{2}\right)\right)\right. \\
& =2 \psi\left(\mathbb{E}^{T}\left(x \circ e^{2}\right)\right)-\psi\left(\mathbb{E}^{T}(x \circ e)\right)-\psi\left(\mathbb{E}^{T}\left(\left(\mathbb{E}^{T} x\right) \circ \mathbb{E}^{T} e^{2}\right)\right) \\
& =2 \psi\left(\mathbb{E}^{T}\left(x \circ e^{2}\right)\right)-\psi\left(\mathbb{E}^{T}(x \circ e)\right)-\psi\left(\mathbb{E}^{T}\left(\left(\mathbb{E}^{T} x\right) \circ e\right)\right) \\
& =2 \psi\left(\mathbb{E}^{T}\left(x \circ e^{2}\right)\right)-2 \psi\left(\mathbb{E}^{T}(x \circ e) .\right.
\end{aligned}
$$

In particular,

$$
\begin{aligned}
{\left[e^{2}, e^{2}\right]_{\psi} } & =2 \psi\left(\mathbb{E}^{T}\left(e^{2} \circ e^{2}\right)\right)-2 \psi\left(\mathbb{E}^{T}\left(e^{2} \circ e\right)\right)=2 \psi\left(\mathbb{E}^{T} e^{4}\right)-2 \psi\left(\mathbb{E}^{T} e^{3}\right) \\
& =2 \psi(e)-2 \psi(e)=0
\end{aligned}
$$

which, by virtue of the Schwarz inequality for the form $[\cdot, \cdot]_{\psi}$, yields

$$
0=\left[x^{*}, e^{2}\right]_{\psi}=2 \psi\left(\mathbb{E}^{T}\left(x \circ e^{2}\right)\right)-2 \psi\left(\mathbb{E}^{T}(x \circ e)\right) .
$$


Since $\psi$ was arbitrary, we obtain

$$
\mathbb{E}^{T}\left(x \circ e^{2}\right)=\mathbb{E}^{T}(x \circ e)
$$

for each $x \in \mathcal{M}$. Consequently, equality (32) takes the form

$$
\begin{aligned}
(e \varphi e)\left(\mathbb{E}^{T} x\right) & =2 \varphi(e \circ(e \circ x))-\varphi(e \circ x) \\
& =2 \varphi\left(\mathbb{E}^{T}(e \circ(e \circ x))\right)-\varphi\left(\mathbb{E}^{T}(e \circ x)\right) \\
& =2 \varphi\left(\mathbb{E}^{T}(e \circ(e \circ x))\right)-\varphi\left(\mathbb{E}^{T}\left(e^{2} \circ x\right)\right. \\
& =\varphi\left(\mathbb{E}^{T}\left(2 e \circ(e \circ x)-e^{2} \circ x\right)\right)=\varphi\left(\mathbb{E}^{T}(e x e)\right) \\
& =\varphi(e x e)=(e \varphi e)(x),
\end{aligned}
$$

showing that $e \varphi e=(e \varphi e) \circ \mathbb{E}^{T}$, i.e. $e \varphi e$ is invariant.

Let now $\left\{e_{i}: i \in I\right\}$ be an arbitrary resolution of identity in $\left(\left(\operatorname{Fix} T^{*}\right)^{h}, \bullet\right)$. Since $\mathbb{E}^{T}(\mathcal{M})=$ Fix $T^{*}$, it follows from $[\mathbf{2 4}$, Lemma 14] that Fix $T$ separates the points of Fix $T^{*}$, and thus $(\operatorname{Fix} T)^{h}$ separates the points of $\left(\operatorname{Fix} T^{*}\right)^{h}$. Consequently, there are $\varphi_{i}^{\prime} \in(\operatorname{Fix} T)^{h}$ such that $\varphi_{i}^{\prime}\left(e_{i}\right) \neq 0$ and considering, if necessary, the positive and negative parts in the Jordan decomposition of $\varphi_{i}^{\prime}$, we may assume that the $\varphi_{i}^{\prime}$ are positive. Put

$$
\varphi_{i}=\frac{e_{i} \varphi_{i}^{\prime} e_{i}}{\varphi_{i}^{\prime}\left(e_{i}\right)} .
$$

Then the $\varphi_{i}$ are invariant normal states such that $\mathrm{s}\left(\varphi_{i}\right) \leqslant e_{i}$. From relation (31), it follows that

$$
\|\psi\|=\sum_{i \in I} \psi\left(e_{i}\right) \geqslant \sum_{i \in I} \varphi_{i}\left(e_{i}\right)=\sum_{i \in I} 1=\operatorname{card} I .
$$

Thus we can have at most $\lfloor\|\psi\|\rfloor$ pairwise orthogonal projections in Fix $T^{*}$. Now the conclusion follows from Proposition 13.

If the $T_{t}^{*}$ are strongly positive, then $\mathrm{Fix} T^{*}$ is a $\mathrm{W}^{*}$-algebra, and since there are at most $\lfloor\|\psi\|\rfloor$ minimal projections in Fix $T^{*}$, we get on account of Lemma 18 that

$$
\operatorname{dim} \operatorname{Fix} T=\operatorname{dim}(\operatorname{Fix} T)^{*}=\operatorname{dim} \operatorname{Fix} T^{*} \leqslant\lfloor\|\psi\|\rfloor^{2} .
$$

Remark 7. It is worth noting that the second part of the thesis of this theorem, concerning the structure of the space $(\operatorname{Fix} T)^{h}$, follows from the assumption

$$
\lim _{t \in G}\left\|\left(T_{t} \varphi-\psi\right)^{+}\right\|=0
$$

for an arbitrary abelian semigroup $G$ since it is a consequence of relation (31) for each invariant normal state $\varphi$. 
Observe that the mean ergodicity of the semigroup $\left(T^{n}\right)$ in the above theorem follows from $[\mathbf{9}$, Theorem 13] and the fact that condition (30) implies that $T$ is asymptotically dominated by the constant operator on $\mathcal{M}_{*}$ which assigns $\psi$ to each element of $\mathcal{M}_{*}$. The same result concerning the connection between asymptotic domination and mean ergodicity in the more general setup of ideally ordered Banach spaces was proved in $[\mathbf{8}$, Theorem 14].

\section{Ergodic theorems for generalised averages}

The following definitions are essentially taken from $[\mathbf{9}]$. Let $\left\{A_{\gamma}: \gamma \in\right.$ $\Gamma\}$ be a generalised average on $G$. The Markov semigroup $\left(T_{t}\right)$ is said to be mean bounded from above (with respect to this average) if there is a nonzero $\psi \in \mathcal{M}_{*}^{+}$such that for each normal state $\varphi$ we have

$$
\lim _{\gamma \in \Gamma}\left\|\left(\left(A_{\gamma}\right)_{T}(\varphi)-\psi\right)^{+}\right\|=0 .
$$

The semigroup is said to be mean bounded from below if there is a nonzero $\psi \in \mathcal{M}_{*}^{+}$such that for each normal state $\varphi$ we have

$$
\lim _{\gamma \in \Gamma}\left\|\left(\left(A_{\gamma}\right)_{T}(\varphi)-\psi\right)^{-}\right\|=0 .
$$

In what follows, we assume that $\left\{A_{\gamma}: \gamma \in \Gamma\right\}$ is a fixed generalised average on $G$ and that $\left(T_{t}\right)$ is a fixed Markov semigroup on $\mathcal{M}_{*}$.

Theorem 20. Let $\left(T_{t}: t \in G\right)$ be mean bounded from above. Then $\left(T_{t}\right)$ is mean ergodic and the fixed-point space $\left(\mathrm{Fix} T^{*}\right)^{h}$ is a JW-algebra which is a finite direct sum of JW-factors of type $\mathrm{I}_{n_{i}}$ with all the $n_{i}$ finite. Moreover, if the $T_{t}^{*}$ are strongly positive, then $\operatorname{Fix} T^{*}$ is a finite dimensional $W^{*}$-algebra.

Proof: From the assumption, it follows that there is a normal positive linear functional $\psi$ such that for each normal state $\varphi$ we have

$$
\lim _{\gamma \in \Gamma}\left\|\left(\left(A_{\gamma}\right)_{T}(\varphi)-\psi\right)^{+}\right\|=0 .
$$

Take an arbitrary normal state $\varphi$. We have

$$
\left(A_{\gamma}\right)_{T}(\varphi) \leqslant \psi+\left(\left(A_{\gamma}\right)_{T}(\varphi)-\psi\right)^{+} .
$$

The $\left(A_{\gamma}\right)_{T}(\varphi)$ are states in the unit ball of $\mathcal{M}^{*}$, so from the weak ${ }^{*}$-compactness of this ball there is a subnet $\left\{A_{\gamma^{\prime}}\right\}$ of $\left\{A_{\gamma}\right\}$ and some state $\widetilde{\varphi} \in$ $\mathcal{M}^{*}$ such that

$$
\left(A_{\gamma^{\prime}}\right)_{T}(\varphi) \underset{\gamma^{\prime}}{\longrightarrow} \widetilde{\varphi} \text { weakly* }
$$


Let $m^{\prime}$ be an invariant mean corresponding to the net $\left\{A_{\gamma^{\prime}}\right\}$. Then

$$
m_{T}^{\prime}(\varphi)=\lim _{\gamma^{\prime}}\left(A_{\gamma^{\prime}}\right)_{T}(\varphi)=\widetilde{\varphi} .
$$

The relation (33) yields

$$
m_{T}^{\prime}(\varphi) \leqslant \psi
$$

and from Lemma 17 it follows that $m_{T}^{\prime}(\varphi) \in \mathcal{M}_{*}$. Hence, on account of Lemma $2,\left(T_{t}\right)$ is mean ergodic.

Let now $\varphi$ be an arbitrary state in Fix $T$. We have

$$
\varphi=\left(A_{\gamma}\right)_{T}(\varphi) \leqslant \psi+(\varphi-\psi)^{+}=\psi .
$$

Let $m$ be an invariant mean. Then

$$
\varphi=m_{T}(\varphi) \leqslant m_{T}(\psi),
$$

and $m_{T}(\psi) \in \mathcal{M}_{*}$ since $\left(T_{t}\right)$ is mean ergodic. Thus we obtain that there is a normal invariant positive linear functional $\widetilde{\psi}=m_{T}(\psi)$ such that $\widetilde{\psi} \geqslant \varphi$ for every state $\varphi$ in $\operatorname{Fix} T$.

The rest of the proof is the same as the final part of the proof of Theorem 14. Let $\left\{e_{i}\right\}$ be an arbitrary resolution of identity in Fix $T^{*}$. We have

$$
\|\widetilde{\psi}\|=\widetilde{\psi}(\mathbb{1})=\sum_{i} \widetilde{\psi}\left(e_{i}\right) .
$$

For each $i$ take a state $\varphi_{i} \in \operatorname{Fix} T$ such that $s\left(\varphi_{i}\right) \leqslant e_{i}$ - the existence of such a state was shown in Theorem 19. We have

$$
1 \leqslant \varphi_{i}\left(e_{i}\right) \leqslant \widetilde{\psi}\left(e_{i}\right) .
$$

Thus

$$
\|\widetilde{\psi}\|=\sum_{i} \widetilde{\psi}\left(e_{i}\right) \geqslant \sum_{i} 1=\operatorname{card}\left\{e_{i}\right\} .
$$

This means that every resolution of identity in Fix $T^{*}$ is finite. Consequently, the conclusion follows from Proposition 13.

Lemma 21. Let $\rho \in \mathcal{M}^{*}$ be singular and let $\omega \in \mathcal{M}_{*}^{h}$. If $\rho \geqslant \omega$, then $\omega \leqslant 0$.

Proof: We have, using the projection $R$,

$$
\omega=R \omega \leqslant R \rho=0 .
$$

The following theorem was obtained in [9, Theorem 3] for a strongly continuous semigroup $\left(T_{t}: t \geqslant 0\right)$ of completely positive operators on $\mathcal{M}_{*}$ and the Cesàro means. The assumption of complete positivity was later shown to be not necessary in [12, Corollary 4.5.5.], where also a more general case of ordered Banach spaces with generating cone was considered. 
Theorem 22. Let $\left(T_{t}: t \in G\right)$ be mean bounded from below. Then

(i) $\left(T_{t}\right)$ is mean ergodic,

(ii) $\operatorname{dim} \operatorname{Fix} T=1$,

(iii) there is a state $\rho \in \operatorname{Fix} T$ such that for each state $\varphi \in \mathcal{M}_{*}$

$$
\text { weak- } \lim _{\gamma \in \Gamma}\left(A_{\gamma}\right)_{T}(\varphi)=\rho \text {. }
$$

Proof: (i) From the assumption, it follows that there is a normal positive linear functional $\psi$ such that for each normal state $\varphi$ we have

$$
\lim _{\gamma \in \Gamma}\left\|\left(\left(A_{\gamma}\right)_{T}(\varphi)-\psi\right)^{-}\right\|=0 .
$$

We are going to show that Fix $T$ separates the points of Fix $T^{*}$. To this end, it is enough to show that for arbitrary $x \in\left(\operatorname{Fix} T^{*}\right)^{h}$ with $\|x\|=1$ there is $\rho \in(\operatorname{Fix} T)^{h}$ for which $\rho(x) \neq 0$. Choose $\varepsilon>0$ such that $\|\psi\|=\psi(\mathbb{1})>\varepsilon$ and take $\varphi \in \mathcal{M}_{*}$ with $\|\varphi\|=1$ satisfying

$$
|\varphi(x)|>1-\varepsilon \text {. }
$$

Let $\varphi=\varphi^{+}-\varphi^{-}$be the Jordan decomposition and assume for the moment that $\varphi^{+} \neq 0 \neq \varphi^{-}$. We have

$$
\left(A_{\gamma}\right)_{T}\left(\frac{\varphi^{+}}{\left\|\varphi^{+}\right\|}\right) \geqslant \psi-\left(\left(A_{\gamma}\right)_{T}\left(\frac{\varphi^{+}}{\left\|\varphi^{+}\right\|}\right)-\psi\right)^{-} .
$$

The $\left(A_{\gamma}\right)_{T}\left(\frac{\varphi^{+}}{\left\|\varphi^{+}\right\|}\right)$are states in the unit ball of $\mathcal{M}^{*}$, so from the weak ${ }^{*}$ compactness of this ball there is a subnet $\left\{\gamma^{\prime}\right\}$ and some state $\widetilde{\rho} \in \mathcal{M}^{*}$ such that

$$
\left(A_{\gamma^{\prime}}\right)_{T}\left(\frac{\varphi^{+}}{\left\|\varphi^{+}\right\|}\right) \underset{\gamma^{\prime}}{\longrightarrow} \widetilde{\rho} \text { weakly* }
$$

Furthermore, we have

$$
\left(A_{\gamma^{\prime}}\right)_{T}\left(\frac{\varphi^{-}}{\left\|\varphi^{-}\right\|}\right) \geqslant \psi-\left(\left(A_{\gamma^{\prime}}\right)_{T}\left(\frac{\varphi^{-}}{\left\|\varphi^{-}\right\|}\right)-\psi\right)^{-}
$$

The $\left(A_{\gamma^{\prime}}\right)_{T}\left(\frac{\varphi^{-}}{\left\|\varphi^{-}\right\|}\right)$are states in the unit ball of $\mathcal{M}^{*}$, so again from the weak ${ }^{*}$-compactness of this ball there is a subnet $\left\{\gamma^{\prime \prime}\right\}$ of $\left\{\gamma^{\prime}\right\}$ and some state $\widetilde{\omega} \in \mathcal{M}^{*}$ such that

$$
\left(A_{\gamma^{\prime \prime}}\right)_{T}\left(\frac{\varphi^{+}}{\left\|\varphi^{+}\right\|}\right) \underset{\gamma^{\prime \prime}}{\longrightarrow} \widetilde{\omega} \quad \text { weakly* }
$$


Let $m$ be an invariant mean corresponding to the net $\left\{A_{\gamma^{\prime \prime}}\right\}$. Then

$$
m_{T}\left(\frac{\varphi^{+}}{\left\|\varphi^{+}\right\|}\right)=\lim _{\gamma^{\prime \prime}}\left(A_{\gamma^{\prime \prime}}\right)_{T}\left(\frac{\varphi^{+}}{\left\|\varphi^{+}\right\|}\right)=\widetilde{\rho}
$$

and

$$
m_{T}\left(\frac{\varphi^{-}}{\| \varphi^{-\|}}\right)=\lim _{\gamma^{\prime \prime}}\left(A_{\gamma^{\prime \prime}}\right)_{T}\left(\frac{\varphi^{-}}{\| \varphi^{-\|}}\right)=\widetilde{\omega} .
$$

Relations (34) and (35) yield

$$
\widetilde{\rho}=m_{T}\left(\frac{\varphi^{+}}{\left\|\varphi^{+}\right\|}\right) \geqslant \psi
$$

and

$$
\widetilde{\omega}=m_{T}\left(\frac{\varphi^{-}}{\left\|\varphi^{-}\right\|}\right) \geqslant \psi \text {. }
$$

Let

$$
\widetilde{\rho}=\widetilde{\rho}_{n}+\widetilde{\rho}_{s} \text { and } \widetilde{\omega}=\widetilde{\omega}_{n}+\widetilde{\omega}_{s}
$$

be decompositions of $\widetilde{\rho}$ and $\widetilde{\omega}$ into the normal and singular parts, respectively. Lemma 21 yields the inequalities $\widetilde{\rho}_{n} \geqslant \psi$ and $\widetilde{\omega}_{n} \geqslant \psi$, and thus

$$
\left\|\widetilde{\rho}_{s}\right\|=\widetilde{\rho}_{s}(\mathbb{1})=1-\widetilde{\rho}_{n}(\mathbb{1}) \leqslant 1-\psi(\mathbb{1})=1-\|\psi\|,
$$

and the same holds for $\left\|\widetilde{\omega}_{s}\right\|$. Since $x \in$ Fix $T^{*}$, it follows that $\varphi(x)=$ $m_{T}(\varphi)(x)$, and we have

$$
\begin{aligned}
1-\varepsilon & <|\varphi(x)|=\left|m_{T}(\varphi)(x)\right| \\
& =\left|\left\|\varphi^{+}\right\| m_{T}\left(\frac{\varphi^{+}}{\left\|\varphi^{+}\right\|}\right)(x)+\left\|\varphi^{-}\right\| m_{T}\left(\frac{\varphi^{-}}{\left\|\varphi^{-}\right\|}\right)(x)\right| \\
& \leqslant\left\|\varphi^{+}\right\|\left|m_{T}\left(\frac{\varphi^{+}}{\left\|\varphi^{+}\right\|}\right)(x)\right|+\left\|\varphi^{-}\right\|\left|m_{T}\left(\frac{\varphi^{-}}{\left\|\varphi^{-}\right\|}\right)(x)\right| \\
& \leqslant\left\|\varphi^{+}\right\|\left(\left|\widetilde{\rho}_{n}(x)\right|+\left|\widetilde{\rho}_{s}(x)\right|\right)+\left\|\varphi^{-}\right\|\left(\left|\widetilde{\omega}_{n}(x)\right|+\left|\widetilde{\omega}_{s}(x)\right|\right) \\
& \leqslant\left\|\varphi^{+}\right\|\left(\left|\widetilde{\rho}_{n}(x)\right|+\left\|\widetilde{\rho}_{s}\right\|\right)+\left\|\varphi^{-}\right\|\left(\left|\widetilde{\omega}_{n}(x)\right|+\left\|\widetilde{\omega}_{s}\right\|\right) \\
& \leqslant\left\|\varphi^{+}\right\|\left(\left|\widetilde{\rho}_{n}(x)\right|+1-\|\psi\|\right)+\left\|\varphi^{-}\right\|\left(\left|\widetilde{\omega}_{n}(x)\right|+1-\|\psi\|\right) \\
& =\left\|\varphi^{+}\right\|\left|\widetilde{\rho}_{n}(x)\right|+\left\|\varphi^{-}\right\|\left|\widetilde{\omega}_{n}(x)\right|+\left(\left\|\varphi^{+}\right\|+\left\|\varphi^{-}\right\|\right)(1-\|\psi\|) \\
& =\left\|\varphi^{+}\right\|\left|\widetilde{\rho}_{n}(x)\right|+\left\|\varphi^{-}\right\|\left|\widetilde{\omega}_{n}(x)\right|+1-\|\psi\|,
\end{aligned}
$$

which yields

$$
\left\|\varphi^{+}\right\|\left|\widetilde{\rho}_{n}(x)\right|+\left\|\varphi^{-}\right\|\left|\widetilde{\omega}_{n}(x)\right|>\|\psi\|-\varepsilon>0 .
$$


Now, it is clear that if, for example, $\varphi^{+}=0$, then we obtain the estimation as above in the form

$$
\left|\widetilde{\omega}_{n}(x)\right|>\|\psi\|-\varepsilon>0,
$$

and similarly if $\varphi^{-}=0$. Consequently, we get that $\widetilde{\rho}_{n}(x) \neq 0$ or $\widetilde{\omega}_{n}(x) \neq$ 0 , and since $\widetilde{\rho}_{n}, \widetilde{\omega}_{n} \in \operatorname{Fix} T$, the separation property follows. On account of $[\mathbf{2 4}$, Lemma 14], we obtain that for a quasi-ergodic projection $\mathbb{E}$, the equality $\mathbb{E}(\mathcal{M})=$ Fix $T^{*}$ holds, which by virtue of Proposition 3 yields the mean ergodicity of $\left(T_{t}\right)$.

(ii) Let $\varphi$ be an arbitrary state in Fix $T$. Then

$$
(\varphi-\psi)^{-}=\left(\left(A_{\gamma}\right)_{T}(\varphi)-\psi\right)^{-} \underset{\gamma}{\longrightarrow} 0 \text { in norm }
$$

which means that $\varphi \geqslant \psi$. Let $\varphi_{1}, \varphi_{2}$ be arbitrary states in Fix $T$. Then $\omega=\varphi_{1}-\varphi_{2} \in \operatorname{Fix} T$ and, for the Jordan decomposition $\omega=\omega^{+}-\omega^{-}$ we have $\omega^{+}, \omega^{-} \in(\operatorname{Fix} T)^{+}$. If $\omega^{+} \neq 0$ and $\omega^{-} \neq 0$, then we would have

$$
\frac{\omega^{+}}{\left\|\omega^{+}\right\|} \geqslant \psi \text { and } \quad \frac{\omega^{-}}{\left\|\omega^{-}\right\|} \geqslant \psi,
$$

which is impossible since $\omega^{+}$and $\omega^{-}$have orthogonal supports. Consequently, $\omega^{+}=0$ or $\omega^{-}=0$ which means that $\varphi_{1} \leqslant \varphi_{2}$ or $\varphi_{1} \geqslant \varphi_{2}$. However, since $\varphi_{1}$ and $\varphi_{2}$ are states, either possibility yields $\varphi_{1}=\varphi_{2}$, showing that there is only one state in $\operatorname{Fix} T$, i.e. $\operatorname{dim} \operatorname{Fix} T=1$.

(iii) Take an arbitrary state $\varphi \in \mathcal{M}_{*}$, and let $\rho \in \mathcal{M}^{*}$ be an arbitrary weak* -limit point of the net $\left\{\left(A_{\gamma}\right)_{T}(\varphi): \gamma \in \Gamma\right\}$. Then

$$
\rho=\text { weak }^{*}-\lim _{\gamma^{\prime}}\left(A_{\gamma^{\prime}}\right)_{T}(\varphi)
$$

for some subnet $\left\{A_{\gamma^{\prime}}\right\}$ of the net $\left\{A_{\gamma}\right\}$. Let $m^{\prime}$ be an invariant mean associated with the net $\left\{A_{\gamma^{\prime}}\right\}$. We have

$$
m_{T}^{\prime}(\varphi)=\rho,
$$

and since $\left(T_{t}\right)$ is mean ergodic, $\rho=m_{T}^{\prime}(\varphi) \in \mathcal{M}_{*}$. Consequently, $\rho \in$ Fix $T$ which means that the limit in (36) is actually a weak limit. Moreover, $\rho$ is a state, and since there is only one state in $\operatorname{Fix} T$, we obtain that

$$
\text { weak- } \lim _{\gamma \in \Gamma}\left(A_{\gamma}\right)_{T}(\varphi)=\rho
$$

for each state $\varphi$, which proves the claim. 
Acknowledgement. The authors are indebted to the referee for a careful reading of the manuscript and numerous remarks and comments which helped to substantially improve the paper. In particular, the short proofs of Lemmas 17 and 21, and the example of the Abel means are due to her/him as well as pointing out a gap in the previous version of Theorem 19. We have also benefited much from her/his knowledge of the literature concerning the problems considered in the paper.

\section{References}

[1] M.-D. Choi And E. G. Effros, Injectivity and operator spaces, J. Functional Analysis 24(2) (1977), 156-209. DOI : 10.1016/0022-1236(77)90052-0.

[2] M. M. DAY, Amenable semigroups, Illinois J. Math. 1(4) (1957), 509-544. DOI : $10.1215 / \mathrm{ijm} / 1255380675$.

[3] N. Dunford and J. T. Schwartz, "Linear Operators. Part I. General Theory", With the assistance of W. G. Bade and R. G. Bartle, Pure and Applied Mathematics 7, Interscience Publishers, Inc., New York; Interscience Publishers, Ltd., London, 1958.

[4] E. G. Effros and E. Størmer, Positive projections and Jordan structure in operator algebras, Math. Scand. 45(1) (1979), 127-138. DOI : 10.7146/math.scand. a-11830.

[5] E. Yu. Emel'yanov, "Non-Spectral Asymptotic Analysis of One-Parameter Operator Semigroups", Operator Theory: Advances and Applications 173, Birkhäuser Verlag, Basel, 2007. DOI : 10.1007/978-3-7643-8114-1.

[6] E. Emel'yanov and N. ERkURSun, Lotz-Räbiger's nets of Markov operators in $L^{1}$-spaces, J. Math. Anal. Appl. 371(2) (2010), 777-783. DOI: 10.1016/j.jmaa. 2010.05.060.

[7] E. Yu. Emel'yanov and M. P. H. WolfF, Quasi constricted linear representations of abelian semigroups on Banach spaces, Math. Nachr. 233/234(1) (2002), 103-110. DOI : 10.1002/1522-2616(200201) 233:1<103: :AID-MANA103>3.0. C0;2Q.

[8] E. Yu. Emel'yanov and M. P. H. Wolff, Positive operators on Banach spaces ordered by strongly normal cones, Positivity 7(1-2) (2003), 3-22. DOI : 10.1023/ A : 1025807816198.

[9] E. Yu. Emel'yanov and M. P. H. Wolff, Asymptotic behavior of Markov semigroups on preduals of von Neumann algebras, J. Math. Anal. Appl. 314(2) (2006), 749-763. DOI : 10.1016/j.jmaa.2005.04.016.

[10] N. ERkurşun Özcan And F. Mukhamedov, Uniform ergodicity of Lotz-Räbiger nets of Markov operators on abstract state spaces, Results Math. 73(1) (2018), Art. 35, 15 pp. DOI : 10.1007/s00025-018-0794-9.

[11] J. GLÜCK AND M. HAASE, Asymptotics of operator semigroups via the semigroup at infinity, Preprint (2018). arXiv:1811.07955.

[12] J. GLüCK ANd M. P. H. WolfF, Long-term analysis of positive operator semigroups via asymptotic domination, Positivity 23(5) (2019), 1113-1146. DOI: 10.1007/s11117-019-00655-7.

[13] F. P. Greenleaf, "Invariant Means on Topological Groups and their Applications", Van Nostrand Mathematical Studies 16, Van Nostrand Reinhold Co., New York-Toronto, Ont.-London, 1969. 
[14] U. Groh, Characterization of positive semigroups on $W^{*}$-algebras, in: "Oneparameter Semigroups of Positive Operators", Lecture Notes in Math. 1184, Springer-Verlag, Berlin, 1986, pp. 376-378. DOI : 10.1007/BFb0074936.

[15] H. Hanche-Olsen and E. Størmer, "Jordan Operator Algebras", Monographs and Studies in Mathematics 21, Pitman (Advanced Publishing Program), Boston, MA, 1984.

[16] N. M. Hugenholtz, $C^{*}$-algebras and statistical mechanics, in: "Operator Algebras and Applications, Part 2" (Kingston, Ont., 1980), Proc. Sympos. Pure Math. 38, Amer. Math. Soc., Providence, R.I., 1982, pp. 407-465.

[17] K. Kielanowicz AND A. ŁuCZAK, Spectral properties of Markov semigroups in von Neumann algebras, J. Math. Anal. Appl. 453(2) (2017), 821-840. DOI: 10. 1016/j.jmaa. 2017.04.029.

[18] J. Komorník, Asymptotic periodicity of Markov and related operators, in: "Dynamics Reported", Expositions in Dynamical Systems (N.S.) 2, Springer, Berlin, 1993, pp. 31-68.

[19] U. Krengel, "Ergodic Theorems", With a supplement by A. Brunel, De Gruyter Studies in Mathematics 6, Walter de Gruyter \& Co., Berlin, 1985. DOI : 10.1515/ 9783110844641.

[20] B. Kümmerer and R. NAGel, Mean ergodic semigroups on $W^{*}$-algebras, Acta Sci. Math. (Szeged) 41(1-2) (1979), 151-159.

[21] A. Lasota, T.-Y. Li, And J. A. Yorke, Asymptotic periodicity of the iterates of Markov operators, Trans. Amer. Math. Soc. 286(2) (1984), 751-764. DOI: 10. $2307 / 1999819$.

[22] A. Lasota and M. C. Mackey, "Probabilistic Properties of Deterministic Systems", Cambridge University Press, Cambridge, 1985. DOI: 10.1017/ CB09780511897474.

[23] A. T. LAU, $W^{*}$-algebras and invariant functionals, Studia Math. 56(3) (1976), 253-261. DOI : $10.4064 / \mathrm{sm}-56-3-253-261$.

[24] A. ŁUCZAK, Invariant states and ergodic dynamical systems on $W^{*}$-algebras, Math. Proc. Cambridge Philos. Soc. 111(1) (1992), 181-192. DOI: 10.1017/ S0305004100075265.

[25] A. ŁuCzAK, Ergodic projection for quantum dynamical semigroups, Internat. J. Theoret. Phys. 34(8) (1995), 1533-1540. DOI : 10.1007/BF00676264.

[26] A. ŁuCZAK, Some ergodic-type theorems for quantum dynamical semigroups, in: "Contributions in Probability" 12, Forum, Udine, 1996, pp. 169-175.

[27] A. ŁuCZAK, Properties of ergodic projection for quantum dynamical semigroups, Internat. J. Theoret. Phys. 37(1) (1998), 555-562. DOI: 10.1023/A: 1026684600279.

[28] A. ŁUCZAK, Range of a contractive strongly positive projection in a $C^{*}$-algebra, Colloq. Math. 140(1) (2015), 87-89. DOI : 10.4064/cm140-1-7.

[29] W. Rudin, "Functional Analysis", McGraw-Hill Series in Higher Mathematics, McGraw-Hill Book Co., New York-Düsseldorf-Johannesburg, 1973.

[30] H. H. Schaefer, "Banach Lattices and Positive Operators", Die Grundlehren der mathematischen Wissenschaften 215, Springer-Verlag, New York-Heidelberg, 1974. DOI : $10.1007 / 978-3-642-65970-6$.

[31] R. Sine, Constricted systems, Rocky Mountain J. Math. 21(4) (1991), 13731383. DOI : $10.1216 / \mathrm{rmjm} / 1181072911$.

[32] M. TAKesaki, "Theory of Operator Algebras I", Springer-Verlag, New YorkHeidelberg, 1979. DOI : 10.1007/978-1-4612-6188-9. 
[33] K. E. Thomsen, Invariant states for positive operator semigroups, Studia Math. 81(3) (1985), 285-291. DOI : 10.4064/sm-81-3-287-291.

Faculty of Mathematics and Computer Science, Łódź University, ul. S. Banacha 22, 90-238 Łódź, Poland

E-mail address: katarzyna.kielanowicz@wmii.uni.lodz.pl

E-mail address: andrzej.luczak@wmii.uni.lodz.pl

Primera versió rebuda el 3 de maig de 2018, darrera versió rebuda el 29 de juliol de 2019. 